\title{
Evaluating Cost-Effectiveness Models for Pharmacologic Interventions in Adults with Heart Failure: A Systematic Literature Review
}

\author{
Gian Luca Di Tanna ${ }^{1} \cdot$ Anna Bychenkova ${ }^{2} \cdot$ Frank O'Neill $^{2} \cdot$ Heidi S. Wirtz ${ }^{3} \cdot$ Paul Miller $^{4} \cdot$ Briain Ó Hartaigh $^{5}$. \\ Gary Globe ${ }^{3}$
}

Published online: 31 December 2018

(c) The Author(s) 2018

\begin{abstract}
Background Heart failure (HF) is a well-recognized public health concern and imposes high economic and societal costs. Decision analytic models exist for evaluating the economic ramifications associated with HF. Despite this, studies that appraise these modelling approaches for augmenting best-practice decisions remain scarce.

Objective Our objective was to conduct a systematic literature review (SLR) of published economic models for the management of HF and describe their general and methodological features.

Methods This SLR employed a combination of relevant search terms associated with HF, which were used in a number of databases, including MEDLINE, Embase, the National Health Service Economic Evaluation Database, Cost-Effectiveness Analysis Registry, ScHARR Health Utilities Database and Cochrane Library Database. A number of model features (i.e. model structure, specification, outcomes assessed, scenario and sensitivity analysis, key model drivers) were extracted and subsequently summarized.

Results Of 64 publications retained, a selection of modelling approaches were identified, including Markov $(n=28)$, trial-based analytic $(n=22)$, discrete-event simulation $(n=6)$, survival analytic $(n=7)$ and decision-tree modelling $(n=1)$ approaches. The bulk of publications employed either a cost-utility $(n=27)$ or cost-effectiveness $(n=36)$ analysis and evaluatedmore than one study outcome, which typically included overall costs $(n=59)$, incremental cost-effectiveness ratios $(n=55)$, life-years gained $(n=48)$ and willingness-to-pay thresholds $(n=37)$. Most publications focused on patients with chronic HF $(n=40)$ and used New York Heart Association (NYHA) disease classifications to categorize patients and determine disease severity. Few $(n=19)$ publications documented the use of hospitalization states for modelling patient outcomes and associated costs. A quality assessment of the included publications revealed most articles demonstrated reasonable methodological value.

Conclusions We identified numerous decision analytic modelling approaches for evaluating the cost effectiveness of pharmacologic treatments in HF. A Markov cohort model approach was most commonly used, and most models relied on NYHA classes as a proxy of HF severity, disease progression and prognosis.
\end{abstract}

Electronic supplementary material The online version of this article (https://doi.org/10.1007/s40273-018-0755-x) contains supplementary material, which is available to authorized users.

Gary Globe

gglobe@amgen.com

1 Economic Modelling Centre of Excellence, Amgen (Europe) $\mathrm{GmbH}$, Rotkreuz, Switzerland

2 Global Health Economics, Amgen Inc, Uxbridge, UK

3 Amgen Inc., One Amgen Center Drive, Thousand Oaks, CA 91320-1799, USA

4 Miller Economics Ltd, Biohub Alderley Park, Alderley Edge, UK

5 Envision Pharma Group, Southport, CT, USA 


\section{Key Points for Decision Makers}

A more comprehensive understanding of the economic models that exist for the management of heart failure is needed.

This systematic literature review identified numerous publications that documented the various decision analytic modelling approaches, with a Markov cohort model the approach most commonly used to assess economic and societal implications in the heart failure setting.

The majority of included studies employed New York Heart Association (NYHA) disease classifications to establish disease severity for modelling purposes.

Further investigation is warranted to help underline the utility of reliable candidates of disease progression and their use in decision analytic models to provide a more precise estimate of the costs incurred while also guiding clinical decision making in the management of heart failure.

\section{Introduction}

Heart failure (HF) has rapidly become a global health concern, with an estimated 26 million people living with this chronic health condition [1-4]. With the bulk of post-industrial nations experiencing a demographic shift, owing mostly to a dramatic growth in the aging population [5], it is not surprising this epidemiologic transition is expected to lead to an increase in the global prevalence and incidence of HF in coming years [2, 6]. Numerous phenotypes for HF exist and are often defined by left-ventricular ejection fraction function [7]. These typically encompass HF with reduced ejection fraction (HFrEF), which reflects systolic dysfunction indicative of impaired contraction in the left ventricle, and $\mathrm{HF}$ with preserved ejection fraction (HFpEF), characterized by abnormal relaxation of the left ventricle [8].

Few treatments specific to patients with HFpEF are available (e.g. diuretics, revascularization for ischaemia, blood pressure control), though evidence indicating improvements in survival based on these pharmacologic therapies within this patient subset is somewhat lacking. A number of pharmacologic treatments are also available for those with HFrEF, including (but not limited to) angiotensin-converting enzyme inhibitors, beta-blockers, angiotensin-II receptor antagonists, mineralocorticoid receptor antagonists and angiotensin receptor inhibitors. Two additional treatments that have recently gained approval for use in the management of HFrEF include sacubitril/valsartan, a first-in-class angiotensin receptor neprilysin inhibitor, and ivabradine, a hyperpolarization-activated cyclic nucleotide-gated channel blocker. In light of these newly approved treatments and those that currently exist in the HF setting, understanding of the economic implications relative to the cost-effectiveness profiles of these pharmacologic regimens needs to improve.

In 2011, Goehler et al. [9] performed a systematic literature review (SLR) of the extant economic modelling approaches available for HF in an effort to examine their usefulness for evaluating health technologies in HF. Several detailed decision models were identified for assessing the various HF technologies, though the bulk of these models differed considerably according to their modelling complexity, approach and underlying assumptions [9]. The majority of these models further relied on New York Heart Association (NYHA) functional classifications as a proxy of disease extent and severity [9].

We conducted an SLR to evaluate published economic models on pharmacologic treatments that reported on health economic outcomes among adults with HF. In particular, we sought to provide an update of the available literature following the article by Goehler et al. [9], which examined data up to June 2010. We further wanted to examine in detail the core modelling specifications and structures as well as key features that were documented in more recently published economic models in the HF patient population.

\section{Methods}

\subsection{Literature Review}

We performed an SLR and identified relevant citations by searching a number of literature databases, including MEDLINE, Embase, the National Health Service Economic Evaluation Database, the Cost-Effectiveness Analysis Registry, the ScHARR Health Utilities Database and Cochrane Library Database using key search terms (Electronic Supplementary Material [ESM] 1). To identify the most up-todate studies pertinent to current clinical practice, the present SLR aimed to include only data published since 1997 (i.e. up until November 2017). The search strategies were confined to all cost-effectiveness studies conducted in human patients with HF. Principal and practical guidelines advocated by the Cochrane Collaboration Handbook [10] and the Centre for Reviews and Dissemination [11] were also employed (where relevant) in an effort to limit any risk of bias and error.

\subsection{Study Eligibility}

This SLR utilized the PICOS (Population, Intervention, Comparator, Outcomes, and Study) design criteria for defining relevant inclusion and exclusion terms. In brief, studies 
were retained for further analysis if they included adult patients who had HF and were aged $\geq 18$ years, provided details related to a pharmacologic treatment for HF and documented any mathematical model of cost-effectiveness specific to patients with HF who received a pharmacologic treatment. We subsequently excluded studies that were observational, experimental, preclinical, pharmacokinetic or pharmacodynamic in nature; a case report or case series (ten or fewer patients); a letter to the editor, opinion piece or review article; or published before 1997.

\subsection{Study Selection}

The titles and abstracts of all retrieved search records were screened to identify any relevant references for inclusion (as per the National Institute for Health and Care Excellence methods guide [12]). Full publications for all retained records were subsequently examined in detail, with a final list of relevant studies compiled thereafter. Both the search and the screening phases were independently conducted by two trained investigators ( $\mathrm{AB}$ and FON). Any disagreements were resolved through further discussion or by consensus with a senior investigator (GDT). Figure 1 presents a PRISMA (Preferred Reporting Items for Systematic Reviews and Meta-Analyses) flow diagram displaying the number of retained studies and a list of excluded studies. In an effort to evaluate the quality of models retrieved from the study selection process, we reviewed good modelling practice methods and employed the CHEERS (Consolidated Health Economic Evaluation Reporting Standards) statement checklist [13, 14].

\subsection{Data Summary}

Data abstracted from the retained publications included study design and population characteristics, model structure and specifications, and specific model outcomes, which are summarized according to the specific research questions, "What is the current availability of cost-effectiveness models for pharmacologic treatments in HF?"; "What are the core model specifications (e.g. types and structures, etc.) of previously published health economic models in HF?"; and "What are the key drivers derived from previously conducted cost-effectiveness analyses in HF?" Data are summarized using text where relevant, or displayed in accompanying tables and figures, as necessary. A brief summary describing the quality of studies based on the CHEERS statement checklist [13] for each research question is also reported. This method aimed to address the appropriateness of the modelling approaches and structures, the quality of reporting, and possible limitations that might have impaired the validity and generalizability of the study findings.
Fig. 1 Flow chart displaying the number of publications included as well as the number of publications that were excluded, with reasons

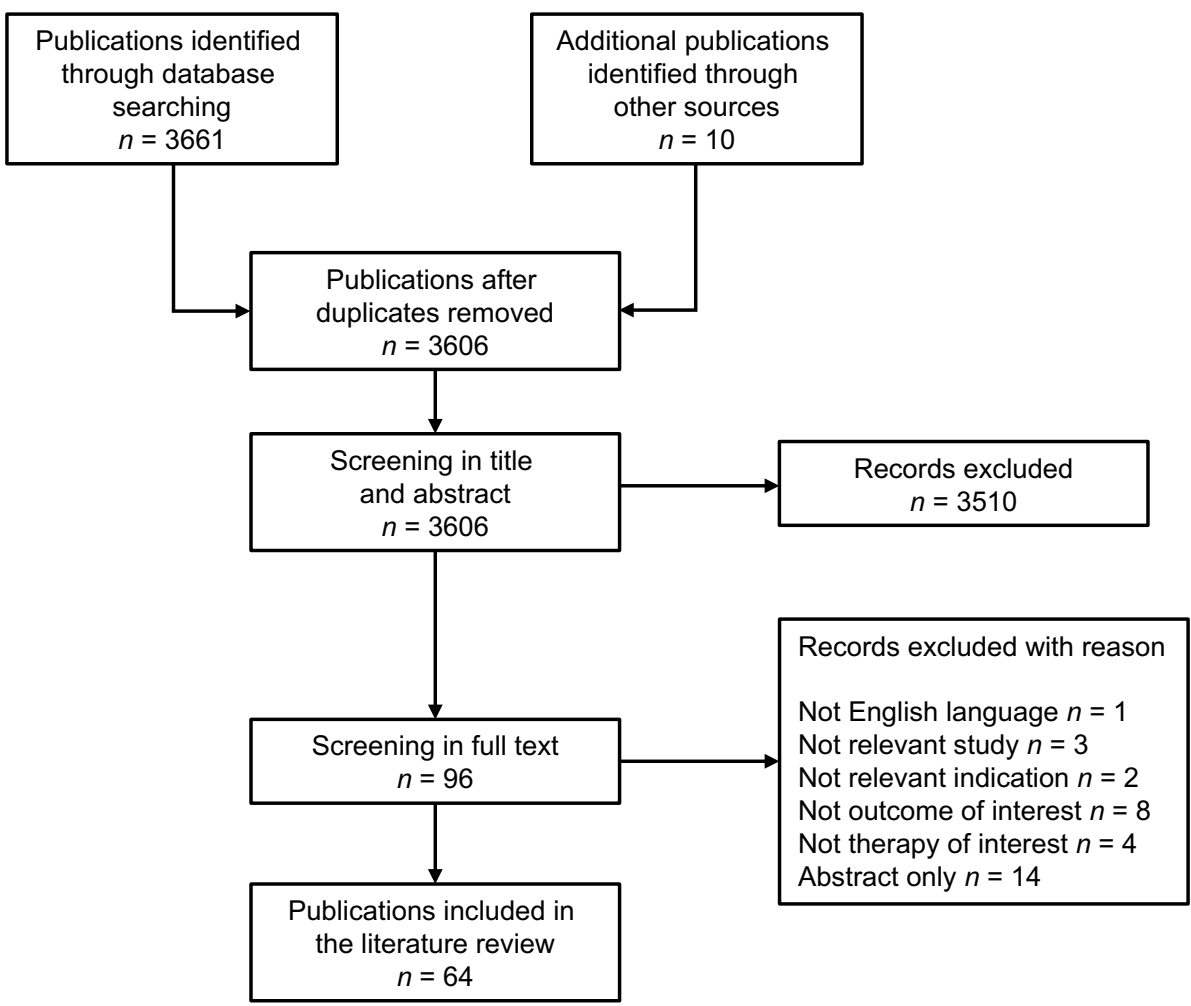




\section{Results}

\subsection{Literature Search}

The original SLR search yielded a total of 3671 citations. Of these, 65 were duplicates that were subsequently removed. A further 3510 articles failed to meet the inclusion criteria based on their titles and abstracts at screening, and an additional 32 publications were excluded for specific reasons as outlined in Fig. 1.

\subsection{Description of Studies}

Overall, 64 articles were suitable for inclusion. The ages of the patient populations ranged from 20 to 93 years. The proportion of men within each patient cohort also varied, with a minimum and maximum of 22 and $89 \%$ documented across publications. Altogether, the geographic regions of the populations in the 64 publications encompassed 15 different countries: the most commonly identified nations included the USA $(n=16$ [25\%]) [15-30], the UK $(n=16$ [25\%]) [31-46] and Canada $(n=5$ [8\%]) [24, 47-50]. A total of $48(75 \%)$ publications included in this review reported their source of funding $[15,16,18,22,24-35,37-42,44,45$, $47,48,50-71]$. Of the included publications, the most commonly reported pharmacologic treatments administered to patients with HF included eplerenone ( $n=12$ [19\%]), ramipril $(n=11[17 \%])$ enalapril $(n=8[13 \%])$ and ivabradine $(n=6[9 \%])$.

\subsection{Overview of Economic Model Structure and Specifications}

\subsubsection{Markov Model Approach}

Most of the publications provided reasonable details in relation to the types of modelling approaches employed (Table 1). The most common modelling approaches used included a Markov cohort model type $(n=28$ [41\%]) [15, $17,19,22,23,26,27,32-34,38-40,44,48,49,51,52$, 58, 66-69, 72-76]. Given the versatility of the Markov model approach in adopting different types of health states patients can potentially experience, this method was further categorized into health state classifications. These typically included an "alive or dead" state $[17,19,32,33,39,51$, 67], NYHA disease classification [15, 23, 52, 58, 68], cardiovascular events [22, 26, 27, 38, 44, 48, 66, 72, 74-76] and hospitalization states [27, 49, 66, 72, 74-76]. Specific Markov model structure was unclear in four studies [34, $40,69,73]$. Seven studies focused on a two-state Markov approach based around the "alive" and "dead" states [17,
19, 32, 33, 39, 51, 67]. Five studies that employed a Markov cohort model approach used the NYHA disease classification system $[15,23,52,58,68]$. Three of these studies [23, $52,58]$ incorporated five health states: one for each NYHA class and a separate one for death. Another publication [68] used four health states according to each of the NYHA classifications to determine health states. A number of methods were employed for modelling according to the NYHA disease classification system, which were based on classifying patients according to their level of HF severity. Some studies employed a constant movement of patients (i.e. belonging to the same health state within the study timeframe) [23, 52], whereas others provided a greater level of detail surrounding transition probabilities [68]. NYHA classifications were generally derived from trial data or from secondary sources when data were unavailable, which may have influenced the estimation of patient ratios within respective NYHA clusters.

Within nine articles that used the Markov model method, HF was often modelled alongside other cardiovascular health states [15, 27, 32, 34, 38, 66, 72, 74, 76]. Aside from $\mathrm{HF}$, other cardiovascular conditions concurrently modelled included myocardial infarction and post cardiovascular events $[34,76]$. Few publications employed hospitalization states with hazard ratios [75] or rate ratios [27] that were applied to transition probabilities in an effort to account for between-treatment effects. One publication [27] adjusted for mortality to increase with age and calibrated mortality rates over the course of the trial to reflect changing probabilities as patients progressed through the model with increasing age. Shorter timeframes of approximately 10 years were implemented in some publications [74], whereas others adopted a lifetime horizon [27, 38, 66, 75, 76]. One study used a random effects meta-analysis to examine the importance of hospitalization as a health state, which was dependent on the number of hospitalizations and death [49]. Other studies associated hospitalization with mortality by implementing survival methods or lifetime tables, linked either directly or independently to hospitalization as a central state of health [75].

\subsubsection{Trial-Based Analytic Approach and Event Simulation}

A number of the retrieved articles followed a trial-based analytic approach $(n=22$ [34\%]) [16, 18, 21, 24, 25, 28-30, 36, 37, 42, 43, 45, 46, 54-57, 59-62]. Some studies [30] analysed clinical trial data with fractional polynomials and piecewise regression to obtain survival hazard functions over time, adjusting for patient characteristics, whereas others [43] employed the life-table method to calculate a gain in life-years, estimating the probability of death using the relative risk of treatment versus placebo from trial data. Certain publications $[46,56]$ extrapolated clinical trial data within 


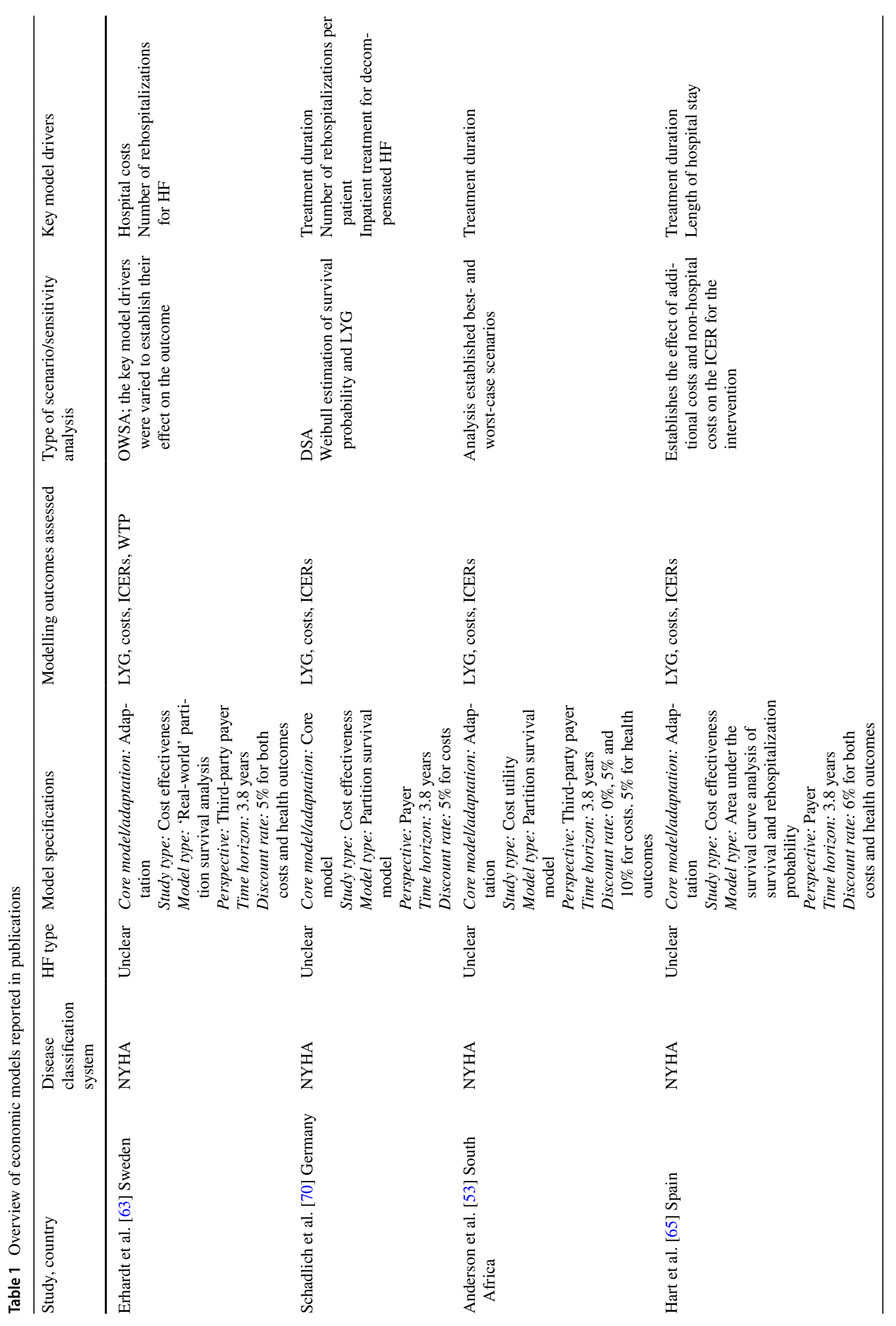




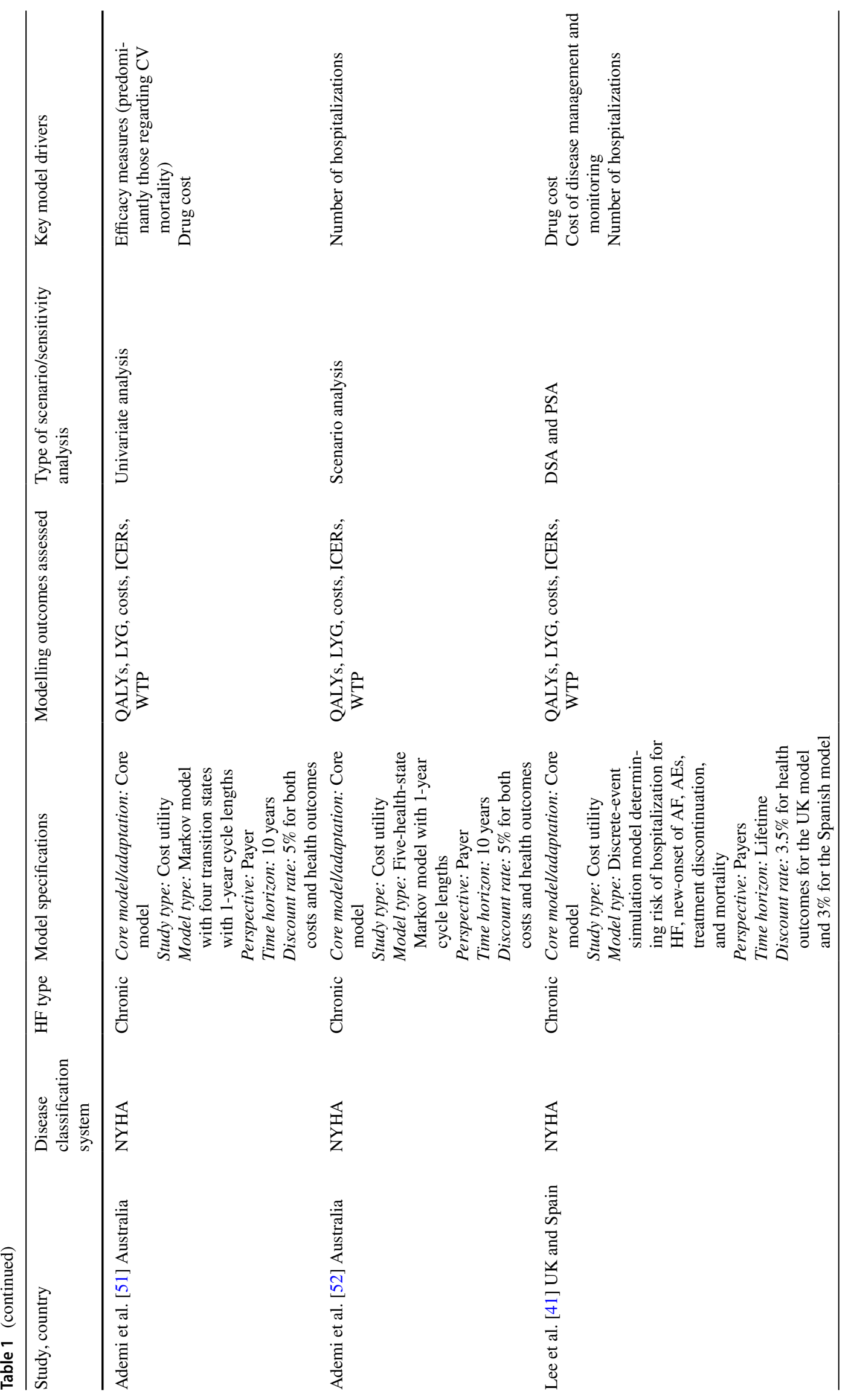




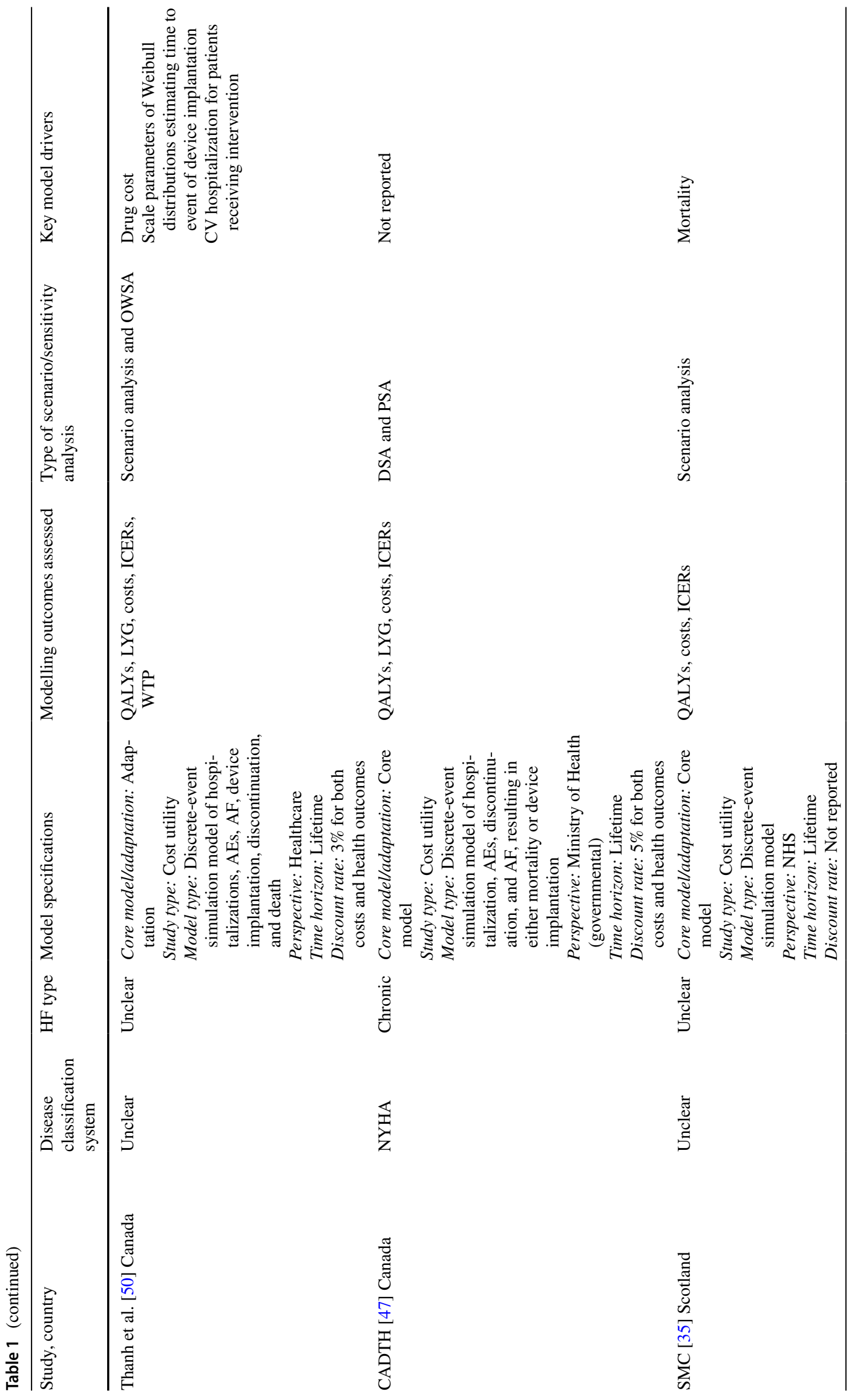




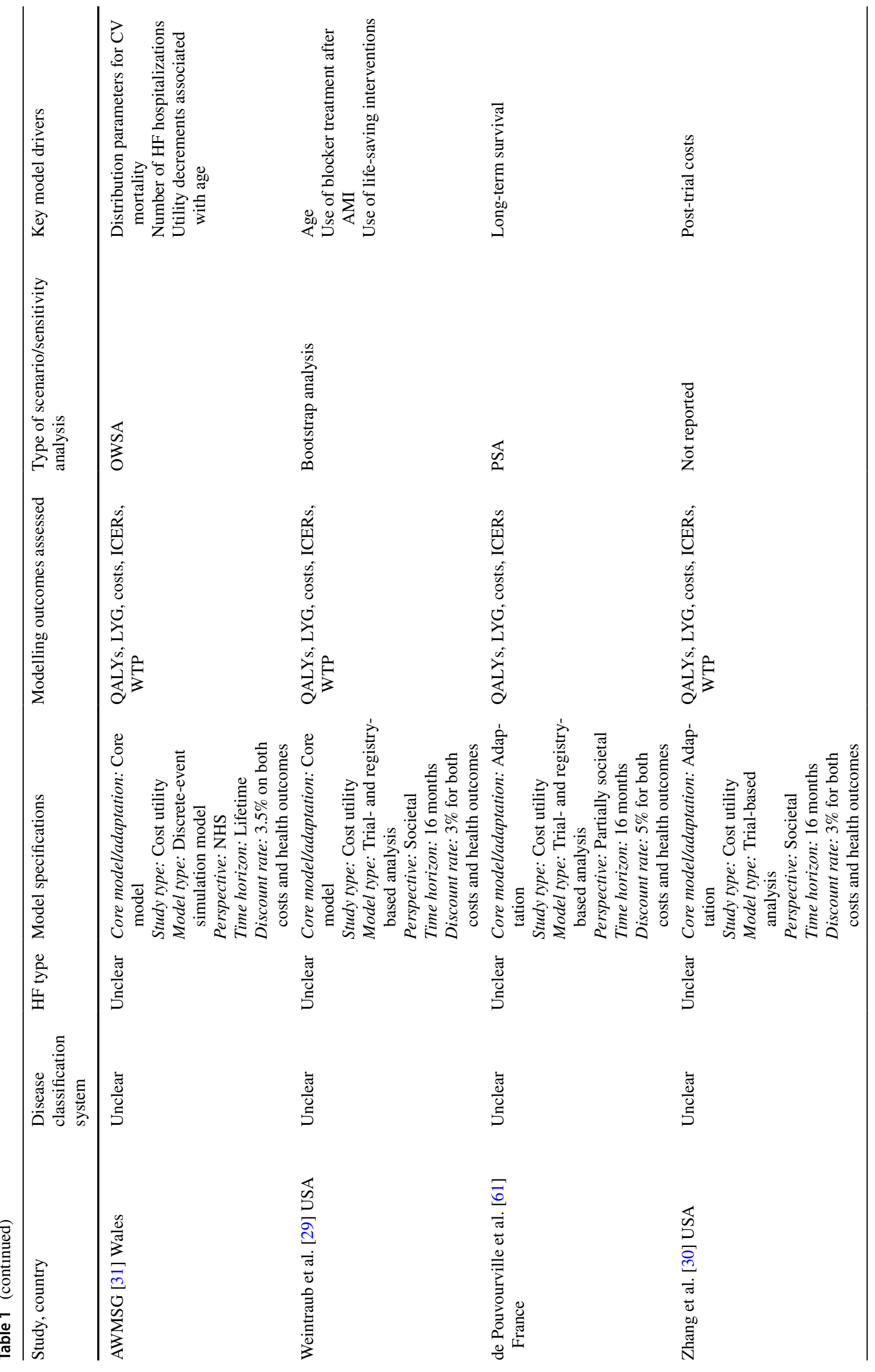




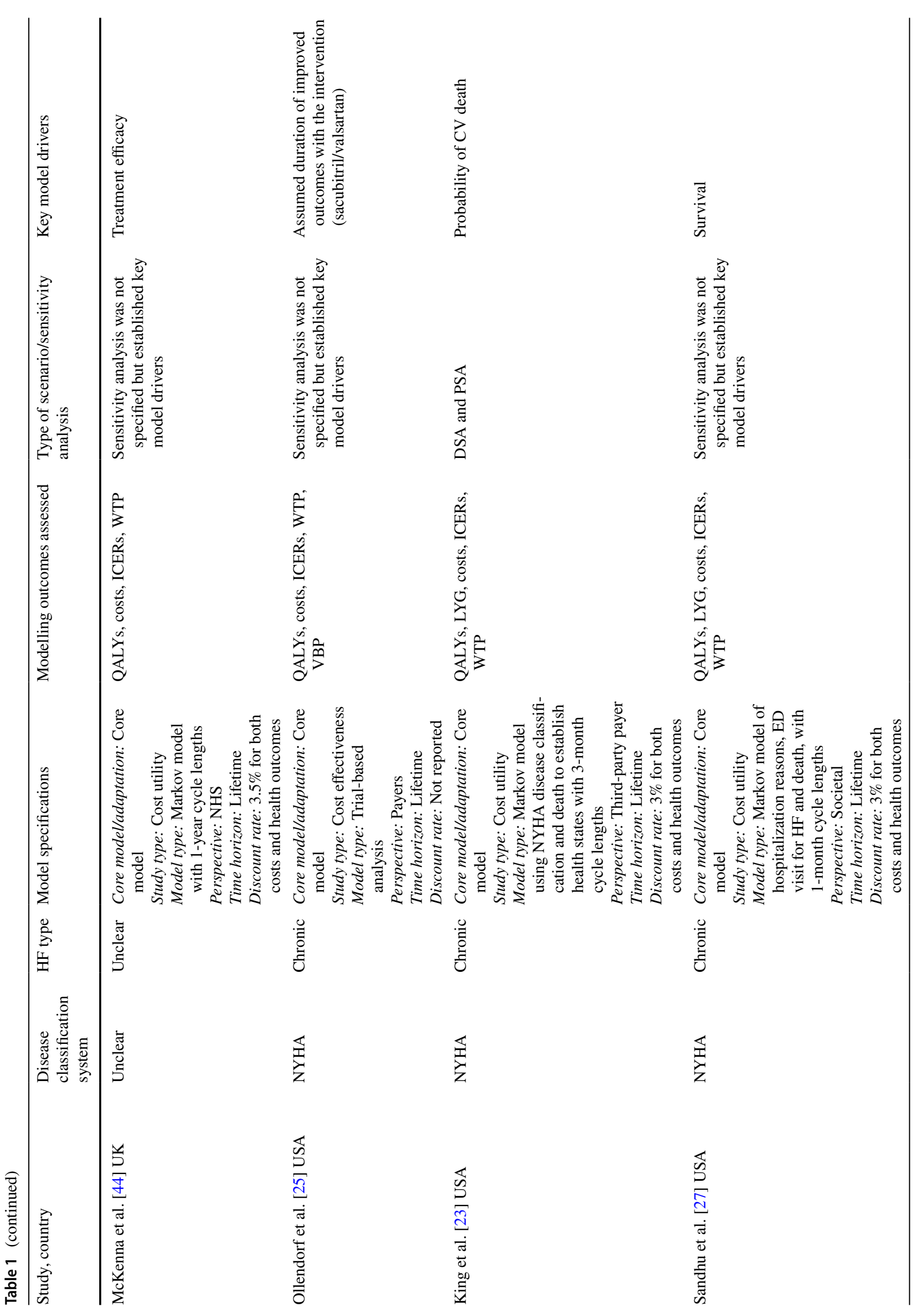




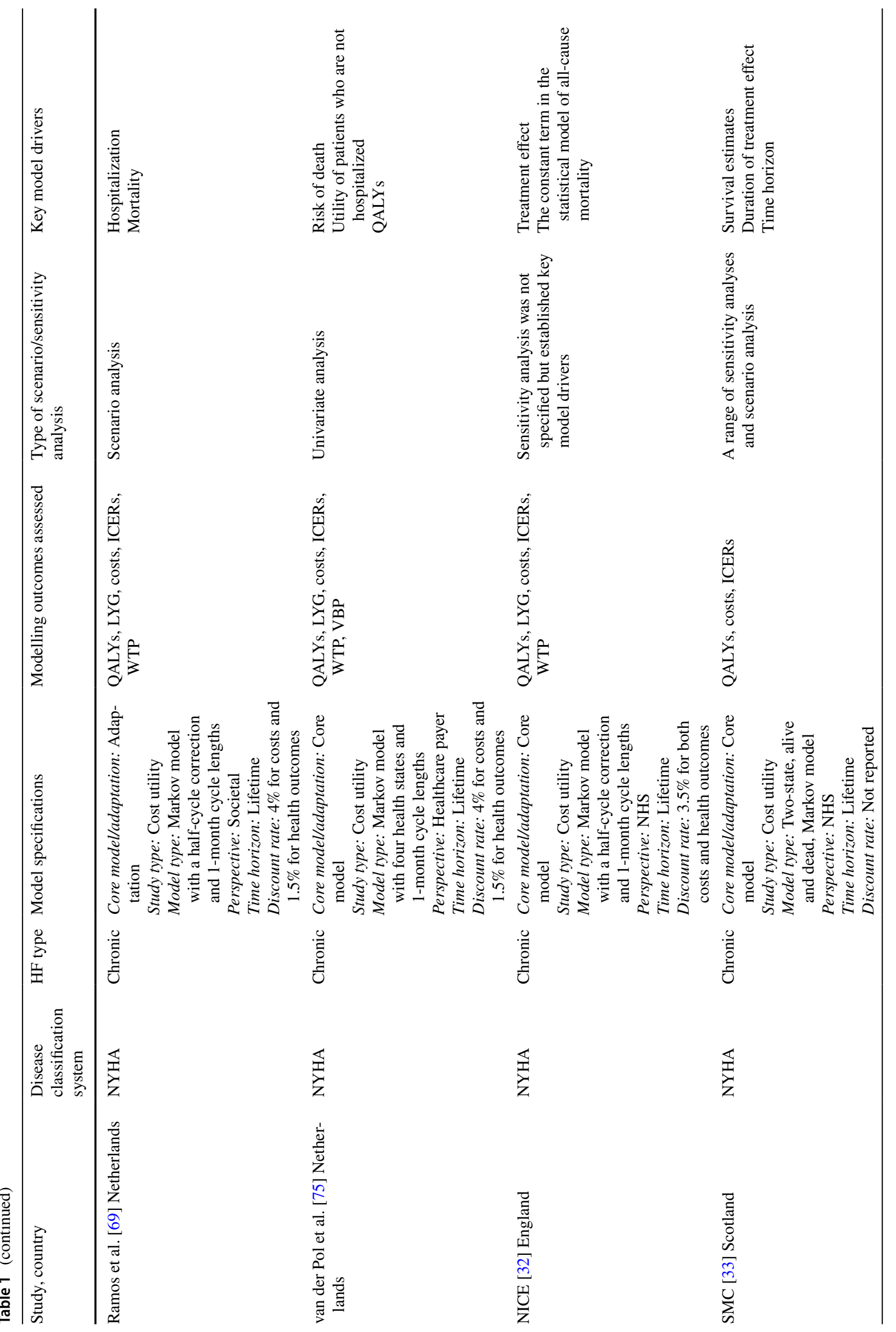




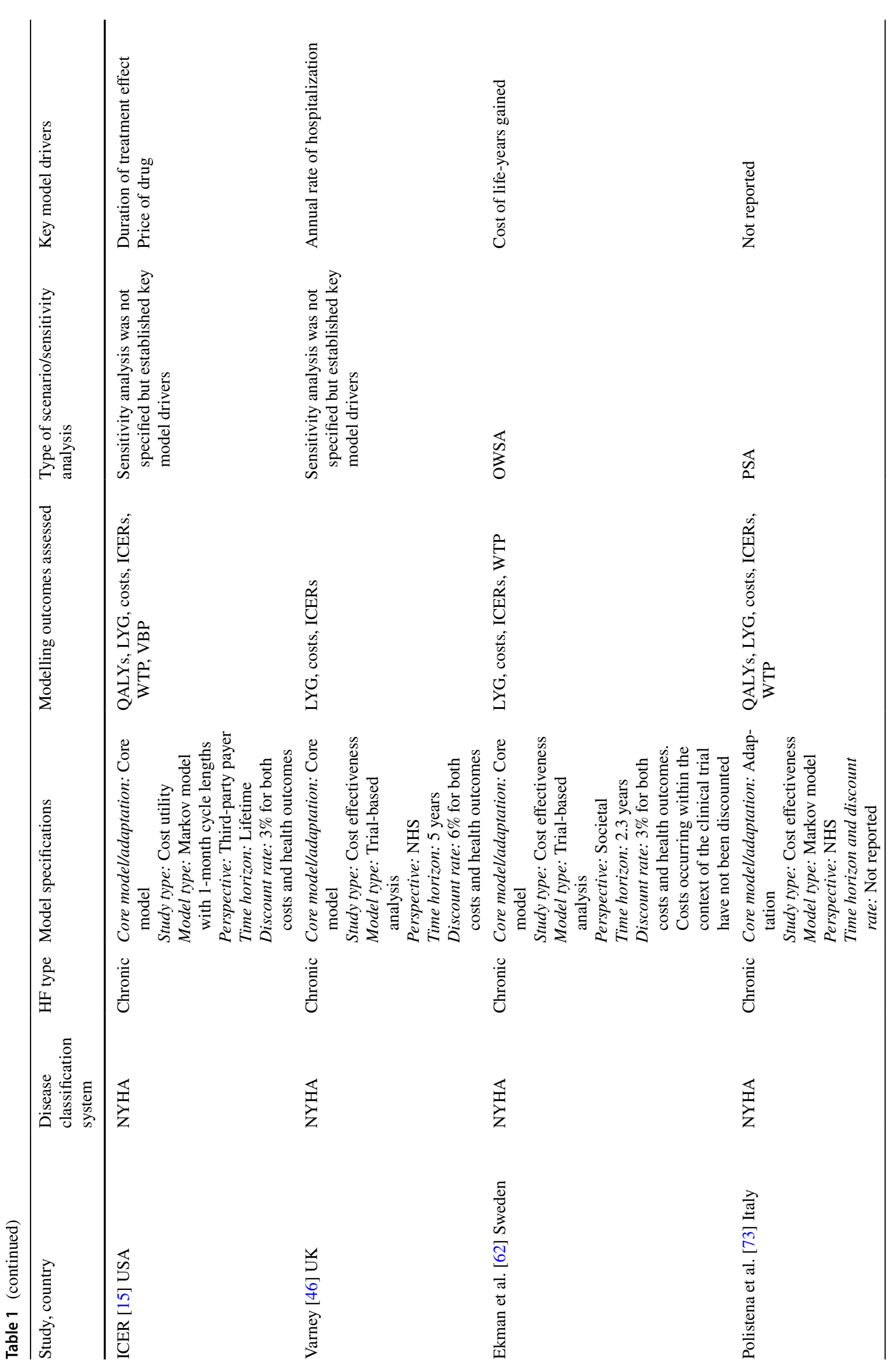




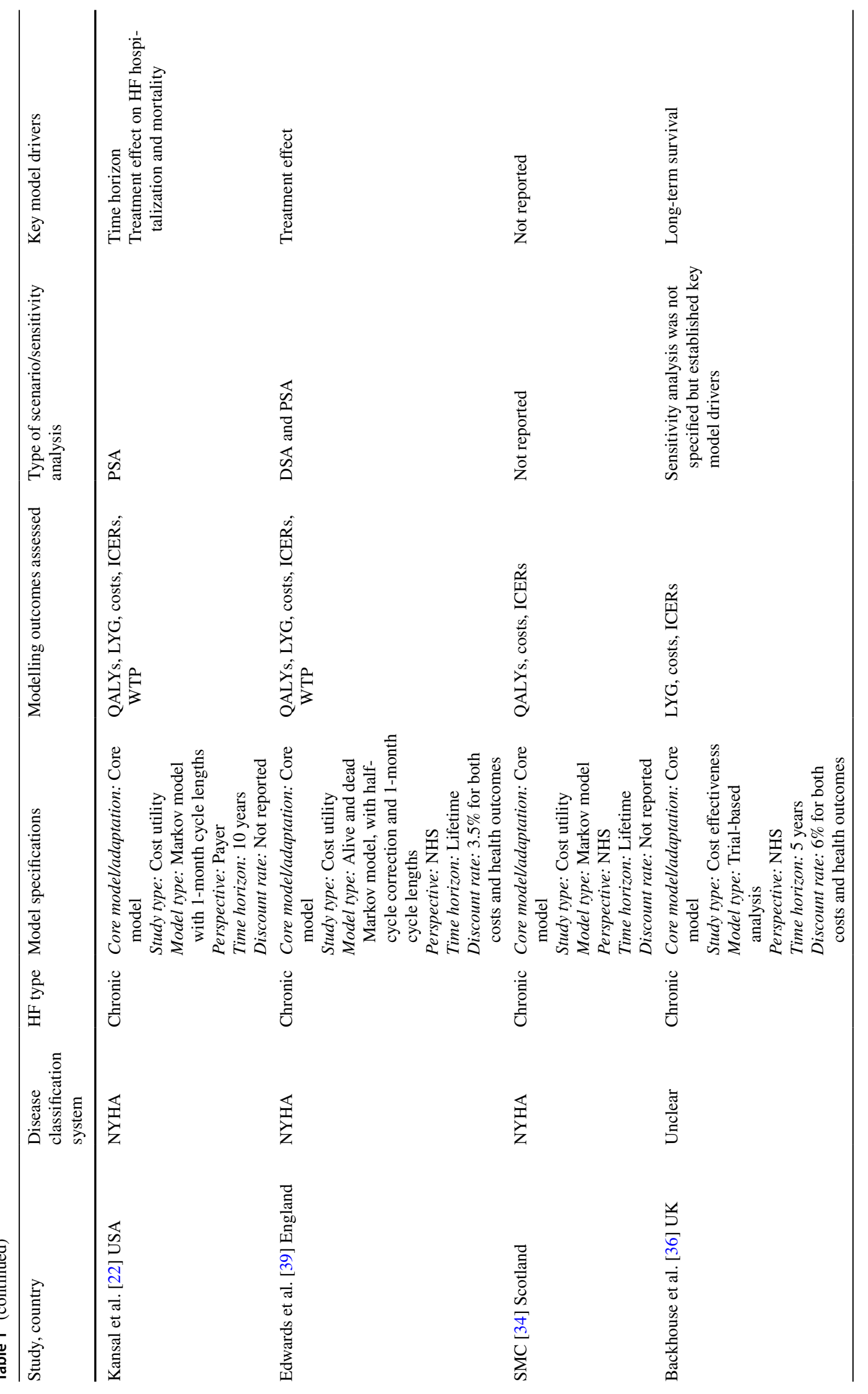




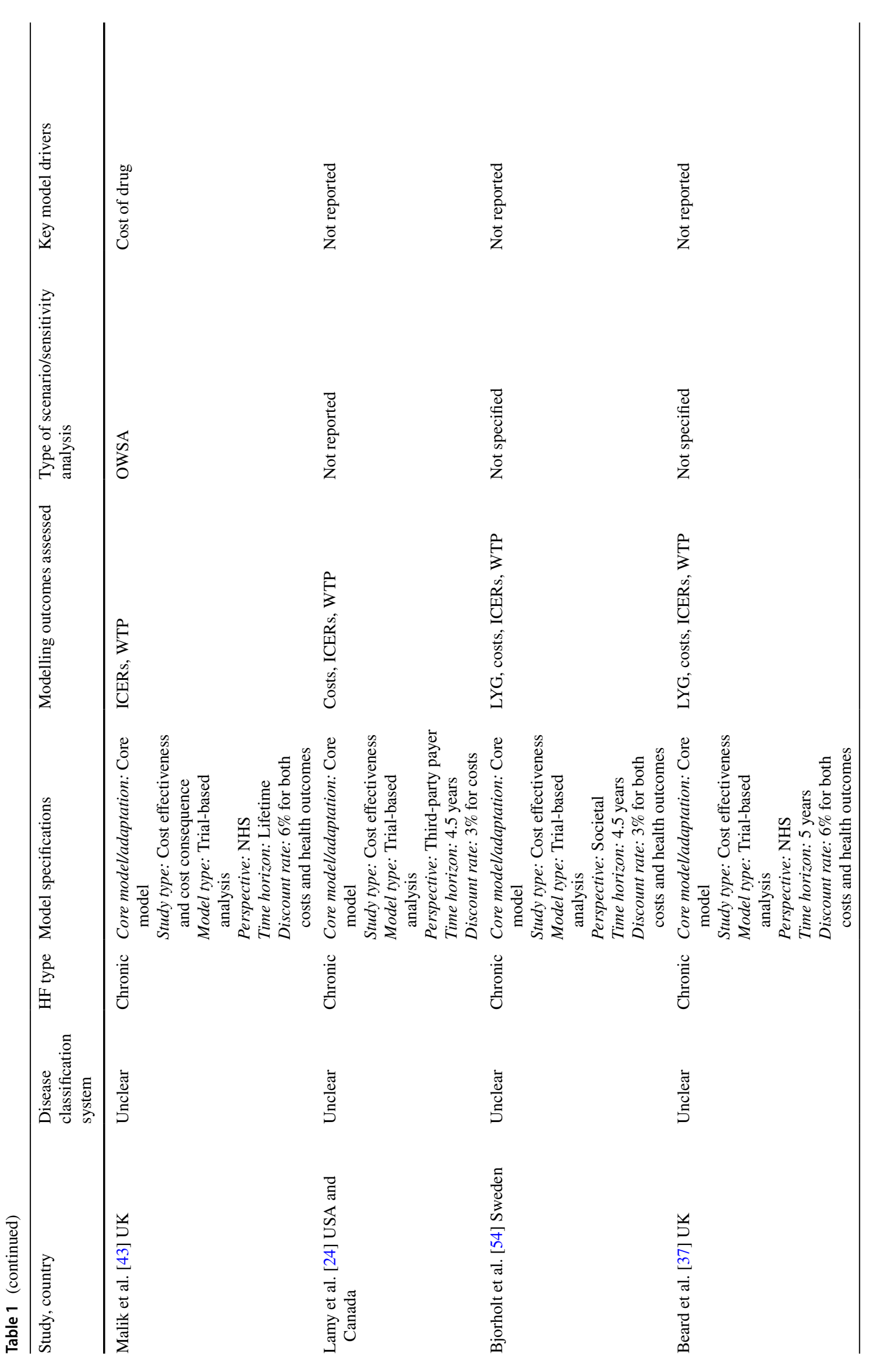




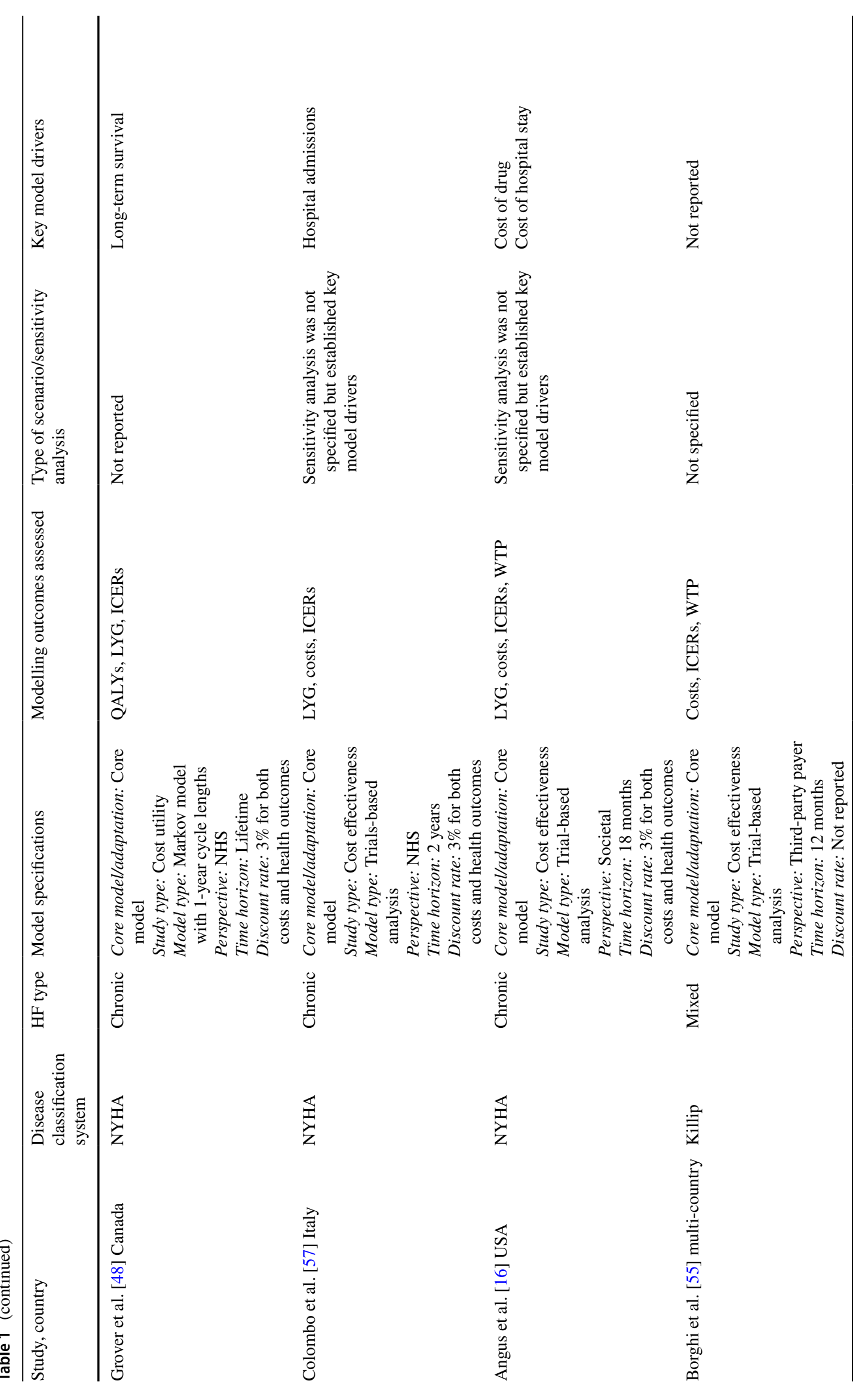




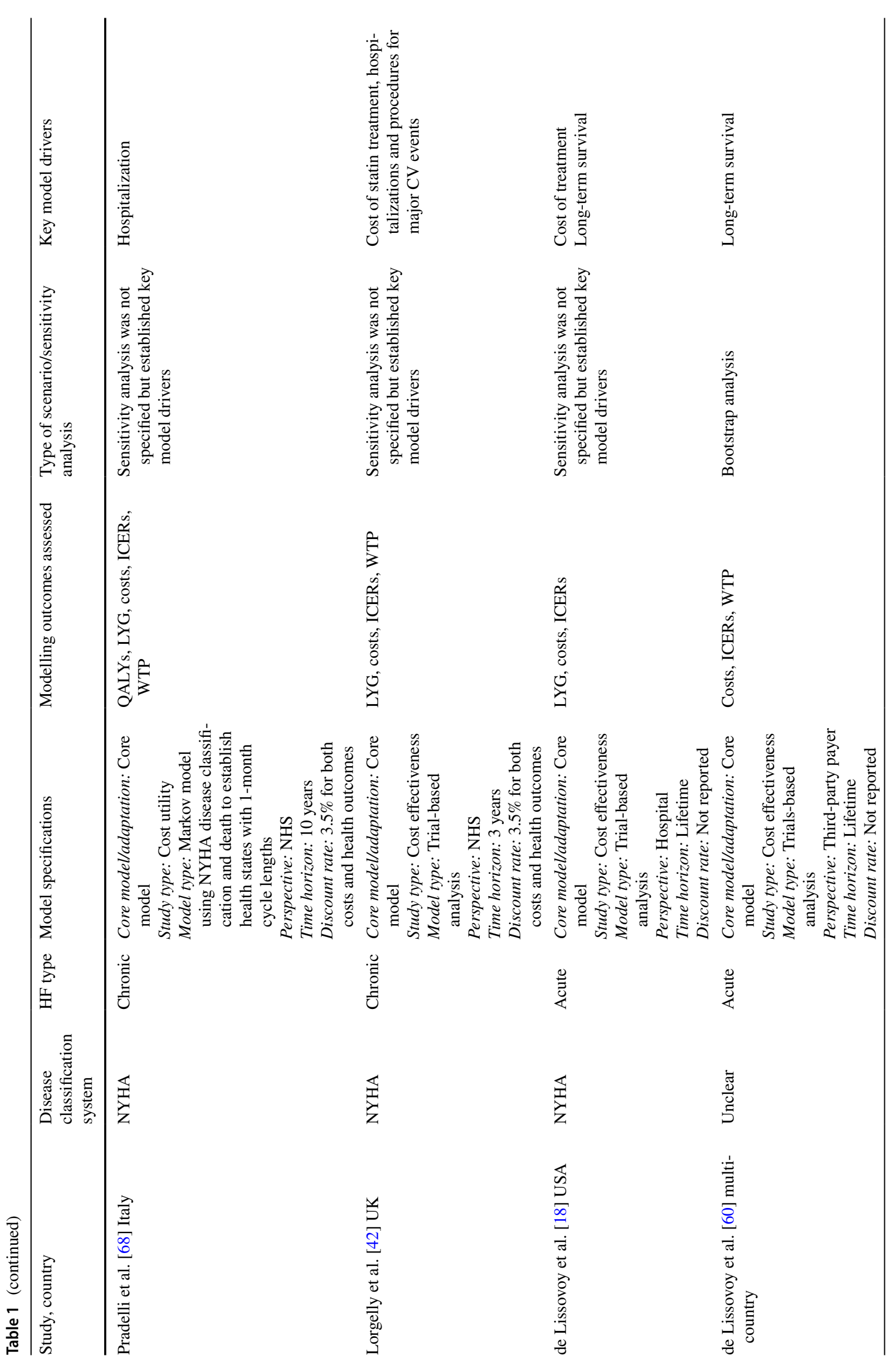




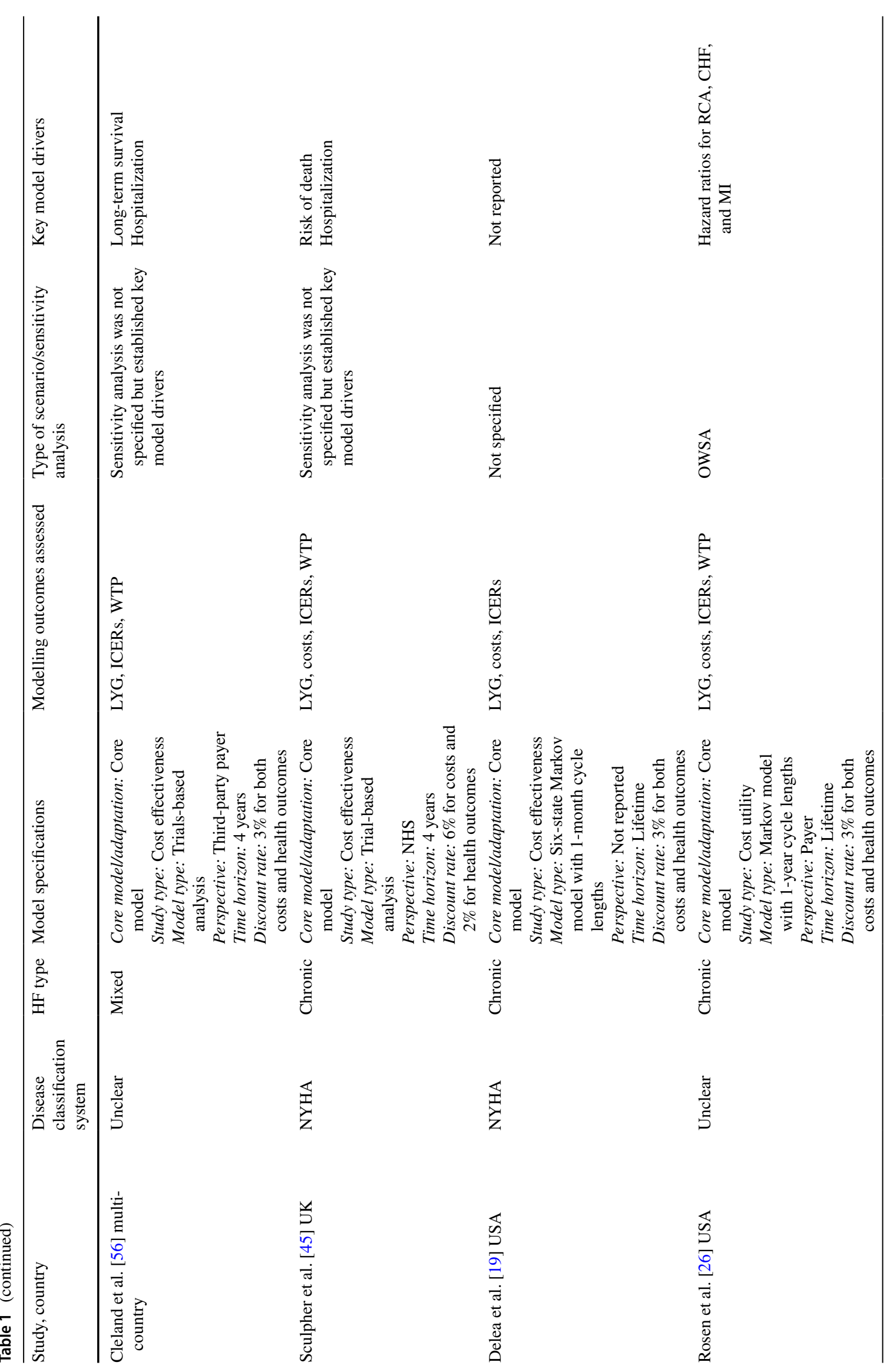




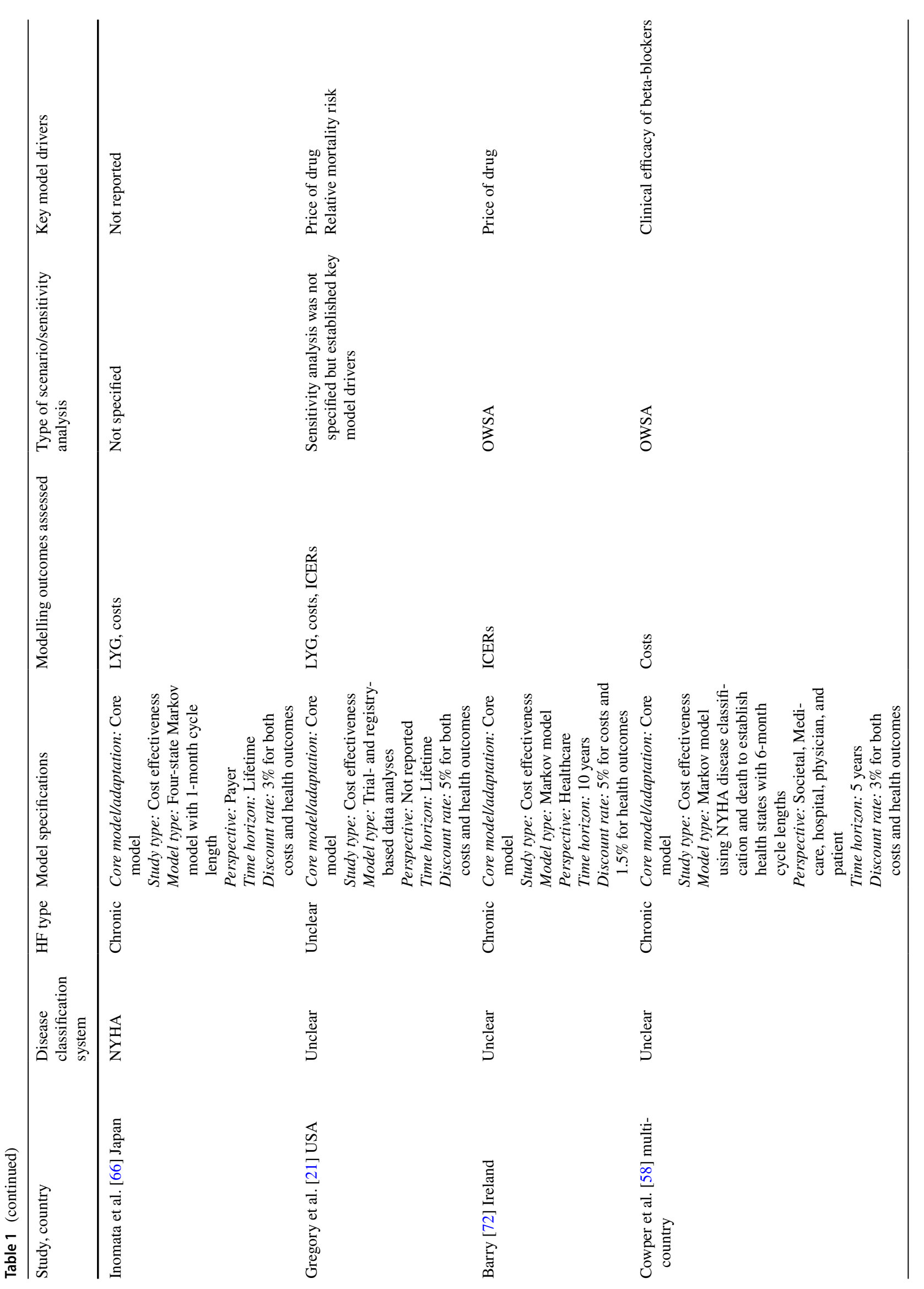




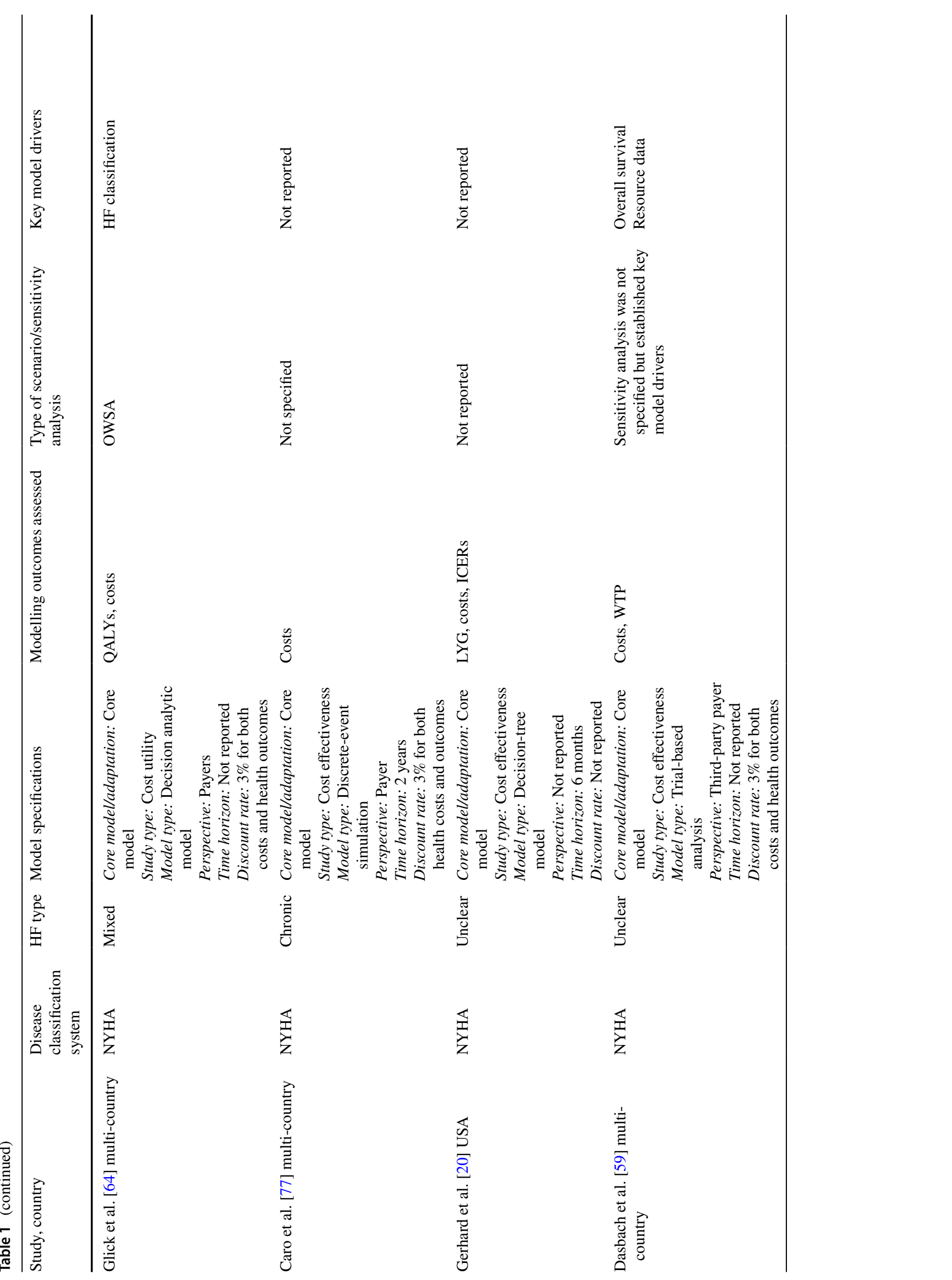




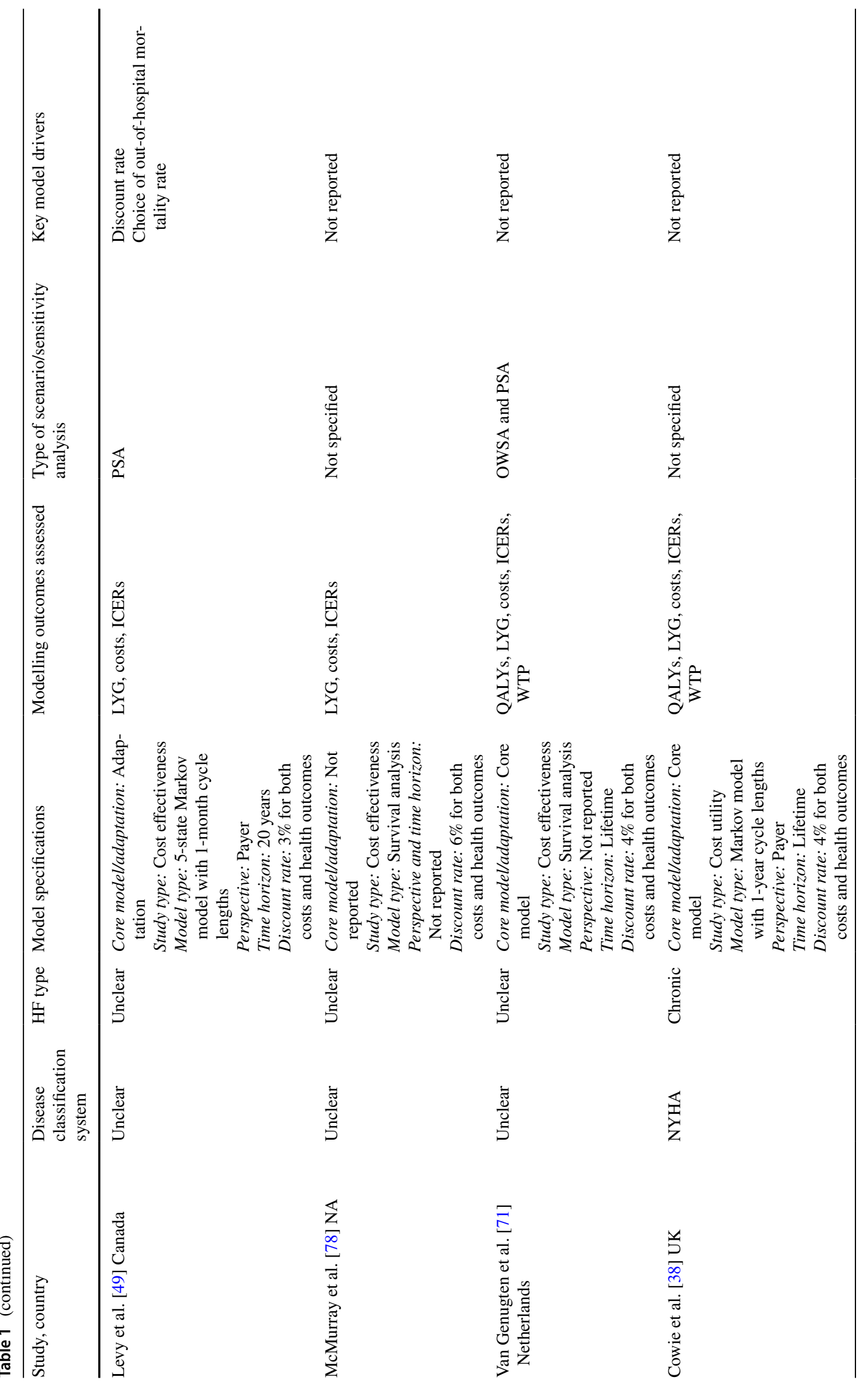




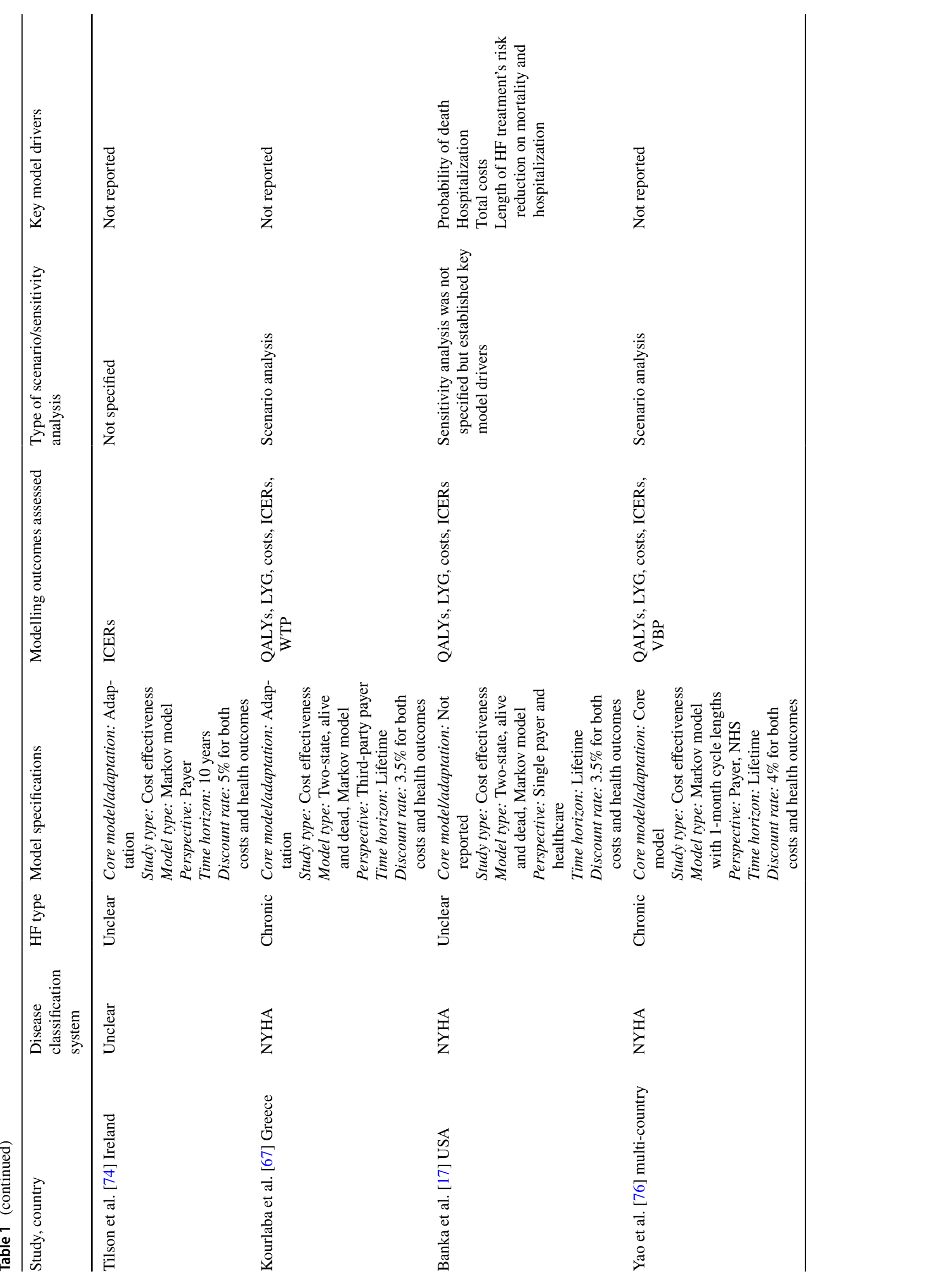




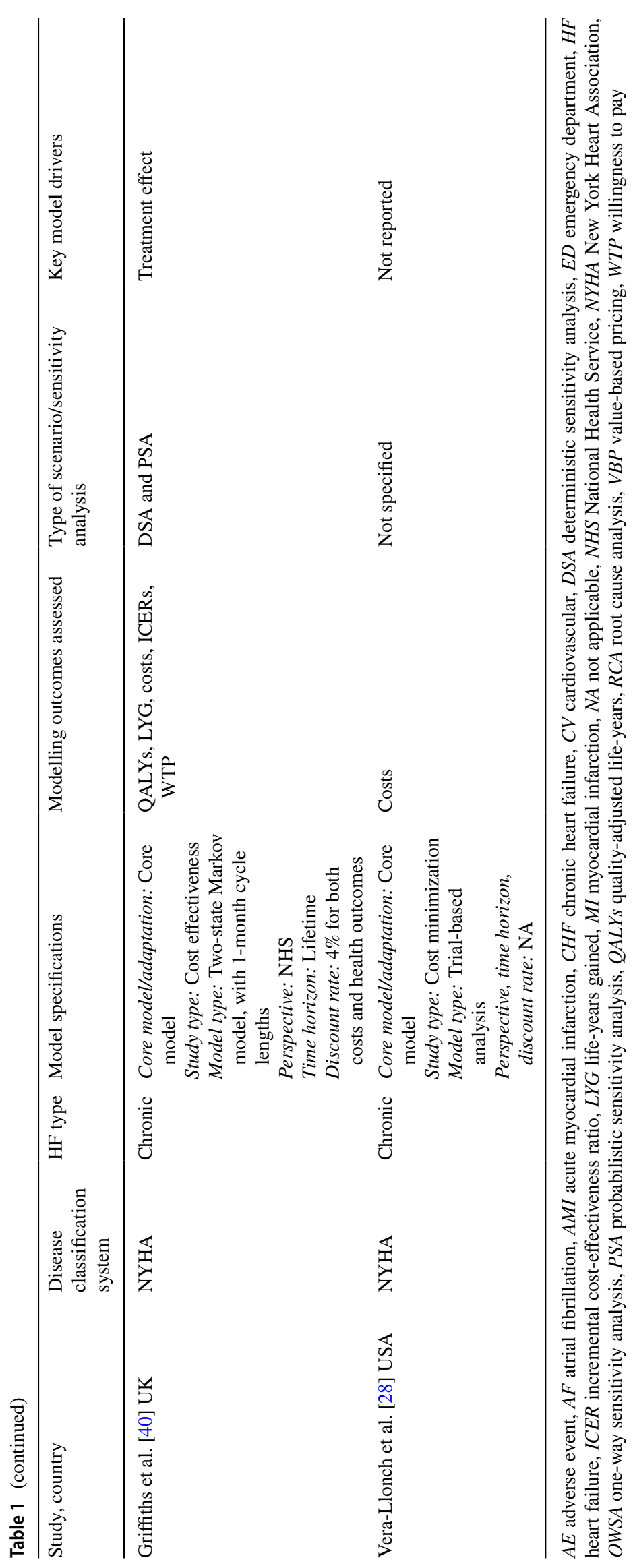


the post-observational period, with others [16] assuming that the treatment efficacy for intervention and placebo declined at the same rate. One publication [30] described the uncertainty for life expectancy values, which was estimated using three external sources. However, given the disparity in population outcomes that were derived from the external sources, these results were considered conservative only [43]. Other approaches that were utilized included a discrete-event simulation $(n=6$ [9\%]) [31, 35, 41, 47, 50, 77]. Two of these studies were derived from health technology assessments $[31,47]$. All six articles relied on the use of clinical trial data $[31,35,41,47,50,77]$, some of which underlined a shorter trial duration $[31,41,50]$ as these trials were terminated early because of the favourable treatment efficacy of the intervention, which in turn, may have influenced model assumptions regarding the initial long-term efficacy assigned to the treatments involved.

\subsubsection{Partition-Type Survival Modelling}

Some articles used a real-world partition-type survival analysis $(n=5[8 \%])[53,63-65,70]$ to evaluate the cost effectiveness of a specific intervention. All publications used the area between Kaplan-Meier curves or time-to-failure survival modelling to estimate differences in life-years gained, hospitalizations and other outcomes between treatment arms. Some indicated that trial data may have underestimated the economic benefit of the intervention by omitting non-study-related physician appointments and laboratory tests $[63,70]$. Information regarding hospital length-of-stay statistics $[63,65]$ or estimated survival rates for the various treatment groups [63] were absent from a number of publications. Most often, differences between treatment arms were captured by the disparity in area under the curves for the probability of first hospitalization as well as the number of life-years gained. Two other publications also used survival analytic models for assessment but did not describe their approach to model structure and specifications [71, 78].

\subsubsection{Decision Tree Modelling}

One publication based their model on the use of a decision tree approach [20]. The absence of Markov nodes made it challenging to assess the overall economic impact, given this study only included a shorter timeline (i.e. maximum duration of 6 months), offering limited insight into the long-term economic implications associated with HF.

\subsection{Health States, Utilities, and Resource Information}

All study types implemented either a cost-utility $(n=27$ [42\%]) or cost-effectiveness approach $(n=36$ [56\%]), with the exception of one publication that used a cost-minimization method [28]. Time horizons tended to vary in duration across publications, ranging from 12 months to a lifetime horizon (Table 1). Most of the models included a health system perspective, with models in a further nine publications based on a societal perspective. The applied discount rates were typically in the range of 3-6\%, depending on where the publication originated (Table 1). A total of 19 studies included information outlining hospitalization states for modelling patient outcomes and associated costs (Fig. 2). The majority $(n=14$ [22\%]) of these health-state types were generally classified as hospitalization.

\subsection{Modelling Outcomes and Sensitivity Analyses}

The bulk of the publications assessed more than one type of study outcome (Table 1). These typically included overall costs $(n=59$ [92\%]), incremental cost-effectiveness ratios (ICERs) $(n=55[86 \%])$, life-years gained $(n=48[75 \%])$ and willingness-to-pay (WTP) thresholds $(n=37$ [58\%]). Figure 3 displays the range of ICERs and WTP thresholds that were obtained according to study outcomes and regions assessed across the retrieved articles. The majority of analyses were evaluated from a US and UK healthcare perspective and used a drug versus placebo approach to establish cost effectiveness. ICERs captured within the UK were typically below the classic threshold of $£ 20,000$ per quality-adjusted life-year (QALY), with similar observations found according to US thresholds (i.e. most below \$US50,000 per QALY). Overall, within the included studies, active treatments (drug vs. drug) yielded higher ICERs than drug versus placebo comparisons (Fig. 3). There were many contributing factors, due to marginal utility gains and higher prices, driving ICER values upwards. It is fair to say that the value of an ICER would be expected to drop with

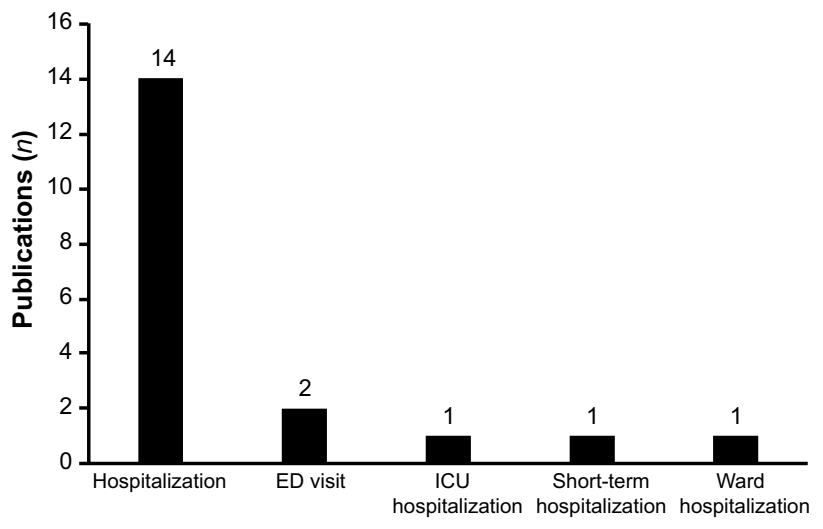

Fig. 2 Hospitalization states utilized by a selection of retrieved studies during model construction for evaluating patient outcomes and associated costs. $E D$ emergency department, $I C U$ intensive care unit 


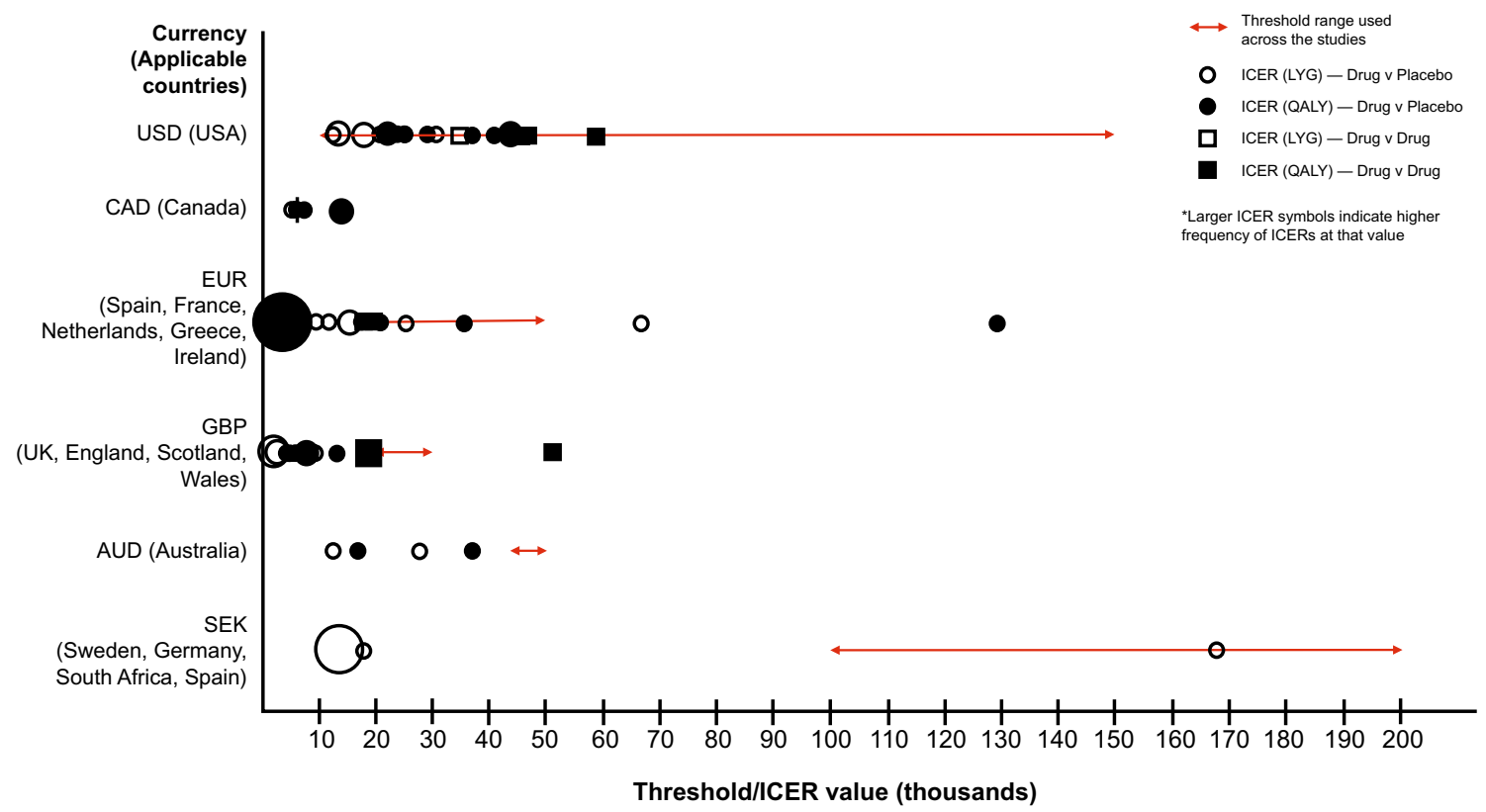

Fig. 3 Frequency and range of incremental cost-effectiveness ratios and willingness-to-pay thresholds according to study outcomes derived from retrieved articles and regions. $A U D$ Australian dollar,
$C A D$ Canadian dollar, EUR Euro, GBP British pound, ICER incremental cost-effectiveness ratio, $L Y G$ life-years gained, $Q A L Y$ qualityadjusted life-year, SEK Swedish krona, USD United States dollar the introduction of new drugs to the market; however, ICER values for drug versus placebo were generally less than drug versus drug comparisons. ICERs also varied widely across countries, partly due to varying approaches within modelling structures and specifications as well as the use and consideration of costs, patient utilities and health gains employed (Fig. 3). Other outcomes that were frequently evaluated included QALYs $(n=32[50 \%])$, with fewer publications $(n=4[6 \%])$ appearing to focus on value-based pricing as a study outcome (Table 1$)$.

A modest number of the retrieved publications performed scenario $(n=4[6 \%])[52,53,67,69]$ and sensitivity analyses, particularly deterministic sensitivity analysis $(n=19$ [30\%]) [23, 26, 31, 39-41, 43, 47, 50, 51, 58, $62-65,70-72,75]$ or probabilistic sensitivity analysis $(n=12$ [19\%]) [22, 23, 29, 39-41, 47, 49, 60, 61, 71, 73]. Although the types of sensitivity analyses were not specified in the remainder of the included publications, most studies were capable of establishing key model drivers. Several components that emerged as key model drivers included the probability of death/survival ( $n=19$ [30\%]) $[17,18,21,23,27,32,33,35,36,45,48,49,51,56$, 59-61, 69, 75], (re)hospitalizations $(n=15$ [23\%]) [17, $31,41,42,45,46,50,52,56,57,63,65,68-70]$, treatment efficacy duration $(n=12$ [19\%]) [15, 17, 22, 32, 33, $39,40,44,53,58,65,70]$ and cost of treatment $(n=9$ [14\%]) $[15,16,18,21,41,43,50,51,72]$.

\subsection{Modelling Disease Type and Classifications}

The majority of published models (40 publications [64\%]) $[15,16,19,22-28,32-34,36-43,45-48,51,52,54,57$, $58,62,66-69,72,73,75-77]$ focused on patient populations that presented with chronic HF, with only two (3\%) $[18,60]$ and three studies $(5 \%)[55,56,64]$ conducting their economic assessment according to patients with acute HF or a mixture of both HF types, respectively (Table 1). Descriptions of patient HF types were unclear in the remaining 19 publications (28\%). With the majority of economic models specific to chronic HF, unsurprisingly, these findings were largely similar to the trends (i.e. for model specifications and structure) documented for the overall number of publications (Table 1). Comparison of the acute HF publications with the overall review findings was not feasible given the paucity of studies that focused solely on acute HF. Likewise, studies in this review that focused on acute and chronic HF (i.e. mixed) concurrently for economic evaluation were also limited. Additional subsets of HF assessed within the retained articles were examined; they included $\operatorname{HFrEF}(n=45[70 \%])$, HFpEF $(n=6[9 \%])$ and both mixed HF subtypes $(n=2$ [3\%]), with the remainder of publications $(n=11[17 \%])$ not reporting a specific HF subtype. Given the lack of differentiation across papers and how they defined patients with $\mathrm{HFrEF}$ and $\mathrm{HFpEF}$, it was also not possible to conduct any comparison according to publications and their patient 
subsets. A total of 41 publications (64\%) [15-17, 19, 20, $22,23,25,27,28,32-34,38-42,45-48,51-53,57,59,60$, $62-70,73,75-77]$ used the NYHA disease classification system to categorize patients and determine the severity of HF, of which 34 (83\%) [15-17, 19, 22, 23, 25, 27, 28, 32-34, $38-42,45-47,51,52,57,59,62,64,66-69,73,75-77]$ focused on patients assigned to NYHA classifications II-III. The observed model structures and specifications within these publications were similar to those found according to the overall number of HF publications (Table 1). Only one publication (2\%) [55] employed the Killip classification system, and definitions of disease classification were unclear in the remainder $(n=22[34 \%])$ (Table 1$)$.

\subsection{Quality of Available Publications}

In total, 63 publications (98\%) were eligible for quality assessment using the CHEERS checklist (Table 2). One study was omitted from quality assessment as only the abstract was presented in English [73]. The majority of checklist items according to the six main category domains were shown to be documented within publications, and most of the included articles demonstrated reasonable methodological quality (Table 2). However, some of the publications only reported on certain items, including "measurement of effectiveness" of data ( $n=31$ [49\%] for both sub-items), "measurement and valuation of performance-based outcomes" $(n=25[40 \%])$, information regarding "estimation of resources and costs" ( $n=22$ [35\%] for item A; $n=35$ [55\%] for item B), as well as "characterizing uncertainty" $(n=22$ [35\%] for item A; $n=36$ [56\%] for item B) and "heterogeneity" $(n=38[60 \%])$ (Table 2).

\section{Discussion}

In this review, we sought to describe the methodological components currently available for use in the economic evaluation of HF, with the intention of guiding future decision analytic models aimed at assessing the cost effectiveness of treatment strategies in the field of HF management. We identified 64 modelling studies, most of which displayed suitable methodological quality. The retrieved models typically employed a Markov or trial-based analytic approach and displayed sufficient details about model perspectives and discount rates. Several recurring outcomes appeared to be commonly assessed within each model, including total costs, ICERs, life-years gained, WTP and QALYs.

Some variability in model structure was evident across the retrieved publications because of the disparity in choice of time horizons used, which ranged from 12 months to a lifetime. We observed a trend away from cost-effectiveness analysis carried out using clinical trial data (or extrapolations from these) towards a modelling-based approach using, for example, Markov modelling. Less than half (i.e. 41\%) of the retrieved publications specified their use of deterministic sensitivity/scenario analysis and/or probabilistic sensitivity analysis [79]. We consider this finding a missed opportunity to assess in detail the parameter uncertainty and the impact of key variables in the cost-effectiveness profiles. Further, although this review identified a number of decision analytic models in the HF setting, collectively, the variability that existed across these models rendered it a challenge to perform a more comprehensive comparison of the retrieved findings. Varying degrees of severity were also found for hospitalization and/or cardiovascular outcomes that were modelled across treatment arms. Some studies tended to use hazard ratios and probabilities from clinical trial data in an effort to estimate the relative probability of HF and hospitalization between treatments. In one publication [39], regression equations employed clinical trial data to determine the treatment effect, whereas other studies used statistical distributions along with survival curves to define clinical efficacies, outcomes and death. Moreover, the rate of survival was frequently derived using time-constant probabilities that differed across treatment arms, health states and/or time horizons $[32,34]$. Other studies tended to calculate mortality as a function of age and sex [68] or by use of survival tables [75]. The complexity of these different methodologies underlines the importance of exploring the most suitable modelling approaches, depending on the research question at hand, for appropriately and reliably conducting economic assessments in the management of HF.

Most studies employed NYHA disease classifications for establishing disease severity among patients for modelling purposes. Yet, it remains questionable whether the predominant use of this instrument (as observed in this review) is appropriate, given limitations around reproducibility, reliability, and clinician's interpretation of what construes "normal" in patients who present with HF [80-82]. Although NYHA functional classification represents a useful clinical measure for defining patient-relevant disease status, alternative classifications may need to be developed to reliably assess disease progression. Our observations support the views of Goehler et al. [9], who documented that most prior models exclusively used NYHA classification as a function of defining disease status, progression and prognosis. From a modelling perspective, the heterogeneity in profiles of patients with HF indicate that sole use of the NYHA instrument to measure disease status is insufficient. Moreover, there is some debate as to how individual clinicians employ the NYHA classifications for evaluating HF severity among patients, with their approaches often considered unclear [82]. Additionally, novel approaches, such as those that implement machine-learning algorithms, would likely extend our understanding of how to select the most suitable 


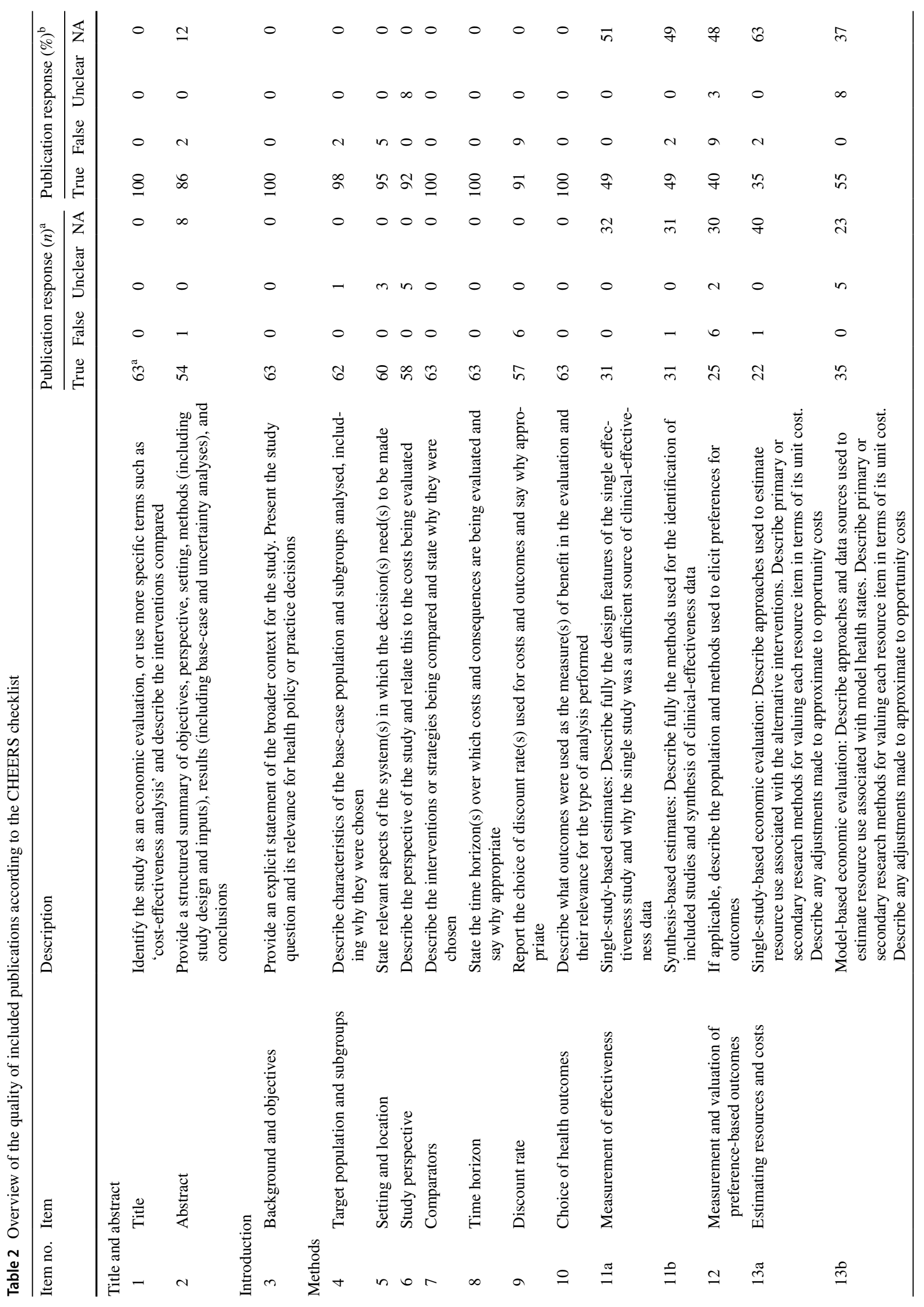




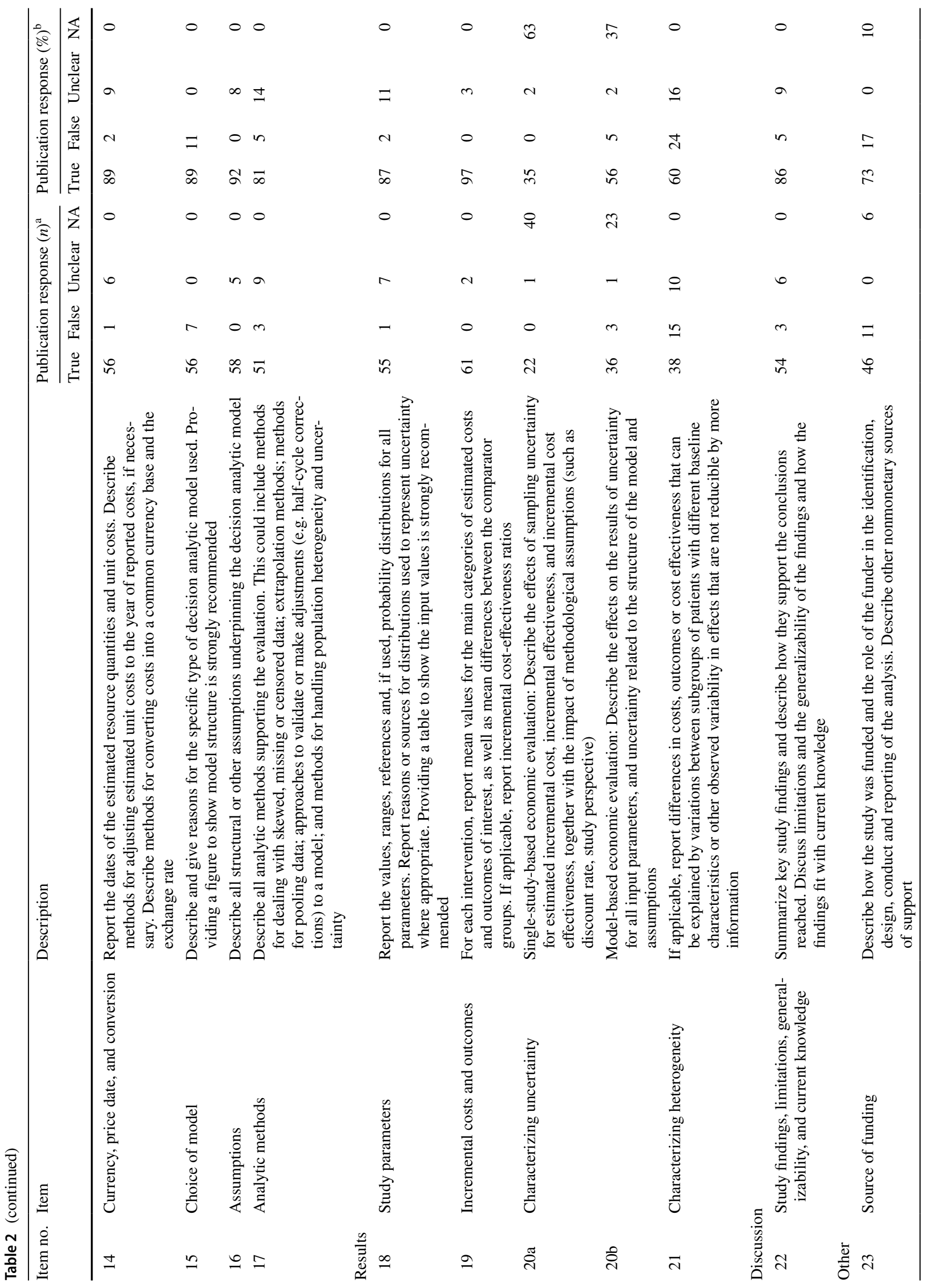


candidate predictors in HF $[83,84]$, through identifying important clusters of risk factors that would facilitate our approach to quantifying disease status in the management and evaluation of HF.

Not all retrieved publications reported details on hospitalizations (e.g. as a health state) when evaluating the costs associated with HF. Of the few studies that provided sufficient information, the majority were classified as general hospitalization. Of those few publications that defined a specific hospitalization type for determining health states, two were classed as emergency department visits, one specified an intensive care unit visit; and two other publications specified a short-term and a ward hospitalization, respectively. Indicators of (re)hospitalization can provide crucial information beyond classification instruments and offer further details about the patient profile, thus proving useful to the modeller when attempting to evaluate the economic and societal implications in HF. Nevertheless, the use of generalized indicators for hospitalization in a model structure should be given careful consideration, alongside any potential for bias or skewing. For example, if hospitalization is only considered as a single Markov state, or if only one hospitalization can occur within one model cycle, a skewing in observations and related costs could occur depending on the actual type and number of hospital visit(s) or actual duration of hospital stays. This is a particular consideration in a chronic condition such as HF, where frequent rehospitalizations are very common $[1,8]$. Although both hospitalization and NYHA disease classifications may offer a more comprehensive assessment of disease status when combined, additional research is undoubtedly needed to help further underline the utility of both measures and their reliability to act as surrogates of HF when attempting to capture disease status, progression and prognosis.

On the background of these reasons, further investigation is warranted to determine more reliable candidates of disease progression that will facilitate forthcoming development of decision analytic models. At minimum, these measures are expected to be readily accessible, straightforward to quantify and have the capacity to provide added value to a model over and above existing parameters (i.e. NYHA class and hospitalization) [32]. Depending on the availability of data and outcomes to be modelled, regression equation modelling such as Poisson and negative binomial regression models could potentially enhance the modelling approach for determining the likelihood that a patient might experience a hospitalization event. Although a number of parametric distributions estimating survival data beyond the measured period provide plausible estimates and an alternative to the Cox model, they do compromise on their restrictions on the shape of the hazard function, often causing an over-estimation in the treatment effect [32]. To overcome this problem, it is 
worth considering more flexible parametric models such as restricted cubic splines to enable modelling of the baseline hazard. This approach offers advantages of greater flexibility in the shape of the hazard function, particularly when compared with the parametric modelling approach.

\subsection{Limitations}

Some limitations inherent to this review need to be emphasized. It was not our intention to offer a comprehensive evaluation of the results of the retrieved studies but rather to provide an overview of similar model features that were employed. It should be borne in mind that each publication differs according to their overall aims, target population considered, health procedures assessed and accessibility to study data. To this end, advocating an optimal modelling approach for use in the HF setting was beyond the scope of this review. Exploring the various economic models according to HF patient subsets, particularly those who presented with either HFrEF or HFpEF, was challenging given the disparity in study sample classifications observed across the retrieved articles. Hence, disentangling the similarities in model structures to identify clear trends in specifics of methodology is beyond the scope of our analysis. In addition, how to best identify key model drivers and specifications according to HF subtypes warrants further investigation. Indeed, we encourage health economics modellers involved in HF treatment assessments to perform value of information analyses (at least expected value of perfect information [EVPI] and expected value of perfect parameter information [EVPPI]) to better evaluate the value of the expected benefit of further investigating uncertain parameters in the decision model [85]. Only models derived from full-text publications were included, and we did not seek direct communication with the corresponding authors of these articles. On this basis, our understanding of the retrieved models is expected to be limited, particularly considering each publication seldom displays all available information because of publication constraints typically administered by journals. As such, we cannot discount the possibility that certain model elements of interest (e.g. as documented in technical modelling reports) may have been overlooked by the present review. We employed a study inclusion window of 20 years for the current analysis. As such, we cannot discount the possibility that other studies deemed suitable for inclusion into this SLR have since been published.

\section{Conclusion}

Numerous decision analytic modelling approaches for evaluating the cost effectiveness of HF treatments have been adopted, with a Markov cohort model proving to be the most commonly used approach. The majority of the models tended to differ in structure as well as health states and frequently relied upon the use of NYHA disease classification as a surrogate of disease severity, progression and prognosis. Recognizing alternative and more succinct approaches for the purpose of defining an optimal model structure would undoubtedly permit for a more precise estimation of the associated costs incurred, while facilitating clinical decision making in the management of HF.

Acknowledgements The authors thank Mitesh Nakum, MSc., and Abigail Shields, MSc. (Curo Payer Evidence, part of the Envision Pharma Group), for their useful insights and assistance and also Benjamin Cohen (University of Southern California) for his preliminary extraction on a short review conducted during his 2017 Summer Internship at Amgen.

Author Contributions All authors adhered to the ICMJE authorship criteria. GL Di Tanna and G Globe planned and designed this study. GL Di Tanna, A Bychenkova and F O'Neill conducted the review, screened the studies and extracted the information. All authors were involved in the data analysis and interpretation, initial drafting of the manuscript, and review and final approval of the manuscript for submission.

\section{Compliance with Ethical Standards}

Funding This study was funded by Amgen, GmbH, Zug, Switzerland. Neither honoraria nor payments were provided for authorship.

Conflict of interest GL Di Tanna, A Bychenkova, H Wirtz, and G Globe are employees of Amgen and may hold corporate stock in Amgen. H Wirtz also holds corporate stock in Teva Pharmaceutical Industries Ltd. F O'Neill was an employee of Amgen until April 2018. P Miller has previously consulted for AstraZeneca, GSK, Pfizer, Novartis, Roche, Chiesi, and Bayer. B Ó Hartaigh was an employee of Curo, part of the Envision Pharma Group, when the study was conducted, who was contracted by Amgen to provide editorial support in the preparation of this manuscript.

Statement of human rights and/or animals For this type of study, formal consent is not required.

Data availability Data sharing is not applicable to this article as no datasets were generated or analysed during the current review.

Open Access This article is distributed under the terms of the Creative Commons Attribution-NonCommercial 4.0 International License (http://creativecommons.org/licenses/by-nc/4.0/), which permits any noncommercial use, distribution, and reproduction in any medium, provided you give appropriate credit to the original author(s) and the source, provide a link to the Creative Commons license, and indicate if changes were made. 


\section{References}

1. Ponikowski P, Anker SD, AlHabib KF, et al. Heart failure: preventing disease and death worldwide. ESC Heart Fail. 2014;1:4-25.

2. Savarese G, Lund LH. Global public health burden of heart failure. Card Fail Rev. 2017;3:7-11.

3. Bui AL, Horwich TB, Fonarow GC. Epidemiology and risk profile of heart failure. Nat Rev Cardiol. 2011;8:30-41.

4. Vos T, Flaxman AD, Naghavi M, et al. Years lived with disability (YLDs) for 1160 sequelae of 289 diseases and injuries 19902010: a systematic analysis for the Global Burden of Disease Study 2010. Lancet. 2012;380:2163-96.

5. Roth GA, Forouzanfar MH, Moran AE, et al. Demographic and epidemiologic drivers of global cardiovascular mortality. N Engl J Med. 2015;372:1333-41.

6. Heidenreich PA, Albert NM, Allen LA, et al. Forecasting the impact of heart failure in the United States: a policy statement from the American Heart Association. Circ Heart Fail. 2013;6:606-19.

7. Yancy CW, Januzzi JL Jr, Allen LA, et al. 2017 ACC Expert Consensus Decision Pathway for Optimization of Heart Failure Treatment: answers to 10 pivotal issues about heart failure with reduced ejection fraction: a report of the American College of Cardiology Task Force on Expert Consensus Decision Pathways. J Am Coll Cardiol. 2018;71:201-30.

8. van Riet EE, Hoes AW, Wagenaar KP, et al. Epidemiology of heart failure: the prevalence of heart failure and ventricular dysfunction in older adults over time. A systematic review. Eur J Heart Fail. 2016;18:242-52.

9. Goehler A, Geisler BP, Manne JM, et al. Decision-analytic models to simulate health outcomes and costs in heart failure: a systematic review. Pharmacoeconomics. 2011;29:753-69.

10. Higgins JPT, Green S, editors. Cochrane handbook for systematic reviews of interventions, version 5.1.0. 2008. https://pdfs.seman ticscholar.org/4b43/91c08c45ebfcd046a53106c97ca09fcdf9f a.pdf. Accessed 31 July 2018.

11. Centre for Reviews and Dissemination. Systematic reviews: CRD's guidance for undertaking reviews in health care. 2009. https://www.york.ac.uk/media/crd/Systematic_Reviews.pdf. Accessed 27 July 2018.

12. National Institute for Health and Care Excellence. Process and methods. In: Guide to the Methods of Technology Appraisal 2013. 2013. London: National Institute for Health and Care Excellence (NICE). https://www.nice.org.uk/process/pmg9/resources/guide -to-the-methods-of-technology-appraisal-2013-pdf-2007975843 781. Accessed 27 July 2018.

13. Husereau D, Drummond M, Petrou S, et al. Consolidated Health Economic Evaluation Reporting Standards (CHEERS) - explanation and elaboration: a report of the ISPOR health economic evaluation publication guidelines good reporting practices task force. Value Health. 2013;16:231-50.

14. Eddy DM, Hollingworth W, Caro JJ, et al. Model transparency and validation: a report of the ISPOR-SMDM Modeling Good Research Practices Task Force-7. Value Health. 2012;15:843-50.

15. Institute for Clinical and Economic Review (ICER). Cardio

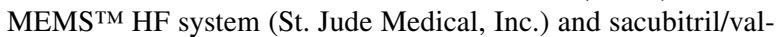
sartan (Entresto ${ }^{\mathrm{TM}}$, Novartis AG) for management of congestive heart failure: effectiveness, value, and value-based price benchmarks. 2016. https://icer-review.org/wp-content/uploads/2016/01/ CHF_Final_Report_120115.pdf. Accessed 22 May 2018.

16. Angus DC, Linde-Zwirble WT, Tam SW, et al. Cost-effectiveness of fixed-dose combination of isosorbide dinitrate and hydralazine therapy for blacks with heart failure. Circulation. 2005;112:3745-53.
17. Banka G, Heidenreich PA, Fonarow GC. Incremental cost-effectiveness of guideline-directed medical therapies for heart failure. J Am Coll Cardiol. 2013;61:1440-6.

18. de Lissovoy G, Fraeman K, Teerlink JR, et al. Hospital costs for treatment of acute heart failure: economic analysis of the REVIVE II study. Eur J Health Econ. 2010;11:185-93.

19. Delea TE, Vera-Llonch M, Richner RE, et al. Cost effectiveness of carvedilol for heart failure. Am J Cardiol. 1999;83:890-6.

20. Gerhard T, Zineh I, Winterstein AG, Hartzema AG. Pharmacoeconomic modeling of nesiritide versus dobutamine for decompensated heart failure. Pharmacotherapy. 2006;26:34-43.

21. Gregory D, Udelson JE, Konstam MA. Economic impact of beta blockade in heart failure. Am J Med. 2001;110(suppl 7A):74s-80s.

22. Kansal AR, Cowie MR, Kielhorn A, et al. Cost-effectiveness of ivabradine for heart failure in the United States. J Am Heart Assoc. 2016;5:e03221.

23. King JB, Shah RU, Bress AP, et al. Cost-effectiveness of sacubitril-valsartan combination therapy compared with enalapril for the treatment of heart failure with reduced ejection fraction. JACC Heart Fail. 2016;4:392-402.

24. Lamy A, Yusuf S, Pogue J, Gafni A. Cost implications of the use of ramipril in high-risk patients based on the Heart Outcomes Prevention Evaluation (HOPE) study. Circulation. 2003;107:960-5.

25. Ollendorf DA, Sandhu AT, Pearson SD. Sacubitril-valsartan for the treatment of heart failure: effectiveness and value. JAMA Intern Med. 2016;176:249-50.

26. Rosen VM, Taylor DC, Parekh H, et al. Cost effectiveness of intensive lipid-lowering treatment for patients with congestive heart failure and coronary heart disease in the US. Pharmacoeconomics. 2010;28:47-60.

27. Sandhu AT, Ollendorf DA, Chapman RH, et al. Cost-effectiveness of sacubitril-valsartan in patients with heart failure with reduced ejection fraction. Ann Intern Med. 2016;165:681-9.

28. Vera-Llonch M, Menzin J, Richner RE, Oster G. Cost-effectiveness results from the US Carvedilol Heart Failure Trials Program. Ann Pharmacother. 2001;35:846-51.

29. Weintraub WS, Zhang Z, Mahoney EM, et al. Cost-effectiveness of eplerenone compared with placebo in patients with myocardial infarction complicated by left ventricular dysfunction and heart failure. Circulation. 2005;111:1106-13.

30. Zhang Z, Mahoney EM, Kolm P, et al. Cost effectiveness of eplerenone in patients with heart failure after acute myocardial infarction who were taking both ACE inhibitors and betablockers: subanalysis of the EPHESUS. Am J Cardiovasc Drugs. 2010;10:55-63.

31. All Wales Medicines Strategy Group (AWMSG). AWMSG secretariat assessment report (full submission). 2016. http://www. awmsg.org/. Accessed 28 May 2018.

32. National Institute for Health and Care Excellence (NICE). Single technology appraisal: sacubitril valsartan for treating heart failure with systolic dysfunction. 2016. https://www.nice.org.uk/guida nce/ta388/documents/committee-papers. Accessed 22 May 2018.

33. Scottish Medicines Consortium (SMC). Sacubitril/valsartan $24 \mathrm{mg} / 26 \mathrm{mg}, 49 \mathrm{mg} / 51 \mathrm{mg}$ and $97 \mathrm{mg} / 103 \mathrm{mg}$ film-coated tablets (Entresto $\left.{ }^{\circledR}\right) .2016$. https://www.scottishmedicines.org.uk/files /advice/sacubitril_valsartan_Entresto_FINAL_February_2016_ for_website.pdf. Accessed 22 May 2018.

34. Scottish Medicines Consortium (SMC). Ivabradine 5 and $7.5 \mathrm{mg}$ film-coated tablets (Procoralan®). 2016. https://www.scottishme dicines.org.uk/files/advice/ivabradine_Procoralan.pdf. Accessed 22 May 2018.

35. Scottish Medicines Consortium (SMC). Eplerenone 25, $50 \mathrm{mg}$ film-coated tablets (Inspra ${ }^{\circledR}$ ). 2012. https://www.scottishmedicin es.org.uk/files/advice/eplerenone_Inspra_FINAL_June_2012_ for_website.pdf. Accessed 22 May 2018. 
36. Backhouse ME, Richter A, Gaffney L. Economic evaluation of ramipril in the treatment of patients at high risk for cardiovascular events. J Med Econ. 2000;3:97-109.

37. Beard SM, Gaffney L, Backhouse ME. An economic evaluation of ramipril in the treatment of patients at high risk for cardiovascular events due to diabetes mellitus. J Med Econ. 2001;4:199-204.

38. Cowie MR, Cure S, Bianic F, et al. Cost-effectiveness of highly purified omega- 3 polyunsaturated fatty acid ethyl esters in the treatment of chronic heart failure: results of Markov modelling in a UK setting. Eur J Heart Fail. 2011;13:681-9.

39. Edwards SJ, Barton S, Nherera L, et al. Ivabradine for the treatment of chronic heart failure: a single technology appraisal. 2012. https://www.nice.org.uk/guidance/ta267/documents/heart-failurechronic-ivabradine-evidence-review-group-report2. Accessed 30 July 2018.

40. Griffiths A, Paracha N, Davies A, et al. The cost effectiveness of ivabradine in the treatment of chronic heart failure from the UK National Health Service perspective. Heart. 2014;100:1031-6.

41. Lee D, Wilson K, Akehurst R, et al. Cost-effectiveness of eplerenone in patients with systolic heart failure and mild symptoms. Heart. 2014;100:1681-7.

42. Lorgelly PK, Briggs AH, Wedel H, et al. An economic evaluation of rosuvastatin treatment in systolic heart failure: evidence from the CORONA trial. Eur J Heart Fail. 2010;12:66-74.

43. Malik IS, Bhatia VK, Kooner JS. Cost effectiveness of ramipril treatment for cardiovascular risk reduction. Heart. 2001;85:539-43

44. McKenna C, Walker S, Lorgelly P, et al. Cost-effectiveness of aldosterone antagonists for the treatment of post-myocardial infarction heart failure. Value Health. 2012;15:420-8.

45. Sculpher MJ, Poole L, Cleland J, et al. Low doses vs. high doses of the angiotensin converting-enzyme inhibitor lisinopril in chronic heart failure a cost-effectiveness analysis based on the Assessment of Treatment with Lisinopril and Survival (ATLAS) study. The ATLAS Study Group. Eur J Heart Fail. 2000;2:447-54.

46. Varney S. A cost-effectiveness analysis of bisoprolol for heart failure. Eur J Heart Fail. 2001;3:365-71.

47. Canadian Agency for Drugs and Technologies in Health (CADTH). CDR pharmacoeconomic review report for Inspra. 2014. https://www.cadth.ca/sites/default/files/cdr/pharmacoec onomic/SR0342_Inspra\%20HF_PE_Report_e.pdf. Accessed 22 May 2018.

48. Grover SA, Coupal L, Lowensteyn I. Estimating the cost effectiveness of ramipril used for specific clinical indications: comparing the outcomes in four clinical trials with a common economic model. Am J Cardiovasc Drugs. 2007;7:441-8.

49. Levy AR, Briggs AH, Demers C, O’Brien BJ. Cost-effectiveness of beta-blocker therapy with metoprolol or with carvedilol for treatment of heart failure in Canada. Am Heart J. 2001;142:537-43.

50. Thanh NX, Ezekowitz JA, Tran DT, Kaul P. Cost effectiveness of eplerenone for the treatment of systolic heart failure with mild symptoms in Alberta, Canada. Am J Cardiovasc Drugs. 2016;16:365-76.

51. Ademi Z, Pasupathi K, Krum H, Liew D. Cost effectiveness of eplerenone in patients with chronic heart failure. Am J Cardiovasc Drugs. 2014;14:209-16.

52. Ademi Z, Pasupathi K, Liew D. Cost-effectiveness of eplerenone compared to usual care in patients with chronic heart failure and NYHA class II symptoms, an Australian perspective. Medicine (Baltimore). 2016;95:e3531.

53. Anderson AN, Moodley I, Kropman K. A South African pharmaco-economic analysis of the Acute Infarction Ramipril Efficacy (AIRE) Study. Cardiovasc J S Afr. 2000;11:89-94.

54. Bjorholt I, Andersson FL, Kahan T, Ostergren J. The cost-effectiveness of ramipril in the treatment of patients at high risk of cardiovascular events: a Swedish sub-study to the HOPE study. J Intern Med. 2002;251:508-17.

55. Borghi C, Ambrosioni E, Omboni S, et al. Cost-effectiveness of zofenopril in patients with left ventricular systolic dysfunction after acute myocardial infarction: a post hoc analysis of SMILE-4. Clinicoecon Outcomes Res. 2013;5:317-25.

56. Cleland JG, Takala A, Apajasalo M, et al. Intravenous levosimendan treatment is cost-effective compared with dobutamine in severe low-output heart failure: an analysis based on the international LIDO trial. Eur J Heart Fail. 2003;5:101-8.

57. Colombo GL, Caruggi M, Ottolini C, Maggioni AP. Candesartan in heart failure: assessment of reduction in mortality and morbidity (CHARM) and resource utilization and costs in Italy. Vasc Health Risk Manag. 2008;4:223-34.

58. Cowper PA, DeLong ER, Whellan DJ, et al. Economic effects of beta-blocker therapy in patients with heart failure. Am J Med. 2004;116:104-11.

59. Dasbach EJ, Rich MW, Segal R, et al. The cost-effectiveness of losartan versus captopril in patients with symptomatic heart failure. Cardiology. 1999;91:189-94.

60. de Lissovoy G, Fraeman K, Salon J, et al. The costs of treating acute heart failure: an economic analysis of the SURVIVE trial. J Med Econ. 2008;11:415-29.

61. de Pouvourville G, Solesse A, Beillat M. Cost-effectiveness analysis of aldosterone blockade with eplerenone in patients with heart failure after acute myocardial infarction in the French context: the EPHESUS study. Arch Cardiovasc Dis. 2008;101:515-21.

62. Ekman M, Zethraeus N, Jonsson B. Cost effectiveness of bisoprolol in the treatment of chronic congestive heart failure in Sweden: analysis using data from the Cardiac Insufficiency Bisoprolol Study II trial. Pharmacoeconomics. 2001;19:901-16.

63. Erhardt L, Ball S, Andersson F, et al. Cost effectiveness in the treatment of heart failure with ramipril. A Swedish substudy of the AIRE study. Acute Infarction Ramipril Efficacy. Pharmacoeconomics. 1997;12:256-66.

64. Glick HA, Orzol SM, Tooley JF, et al. Economic evaluation of the randomized aldactone evaluation study (RALES): treatment of patients with severe heart failure. Cardiovasc Drugs Ther. 2002;16:53-9.

65. Hart WM, Rubio-Terres C, Pajuelo F, Gonzalez Juanatey JR. Cost-effectiveness of the treatment of heart failure with ramipril: a Spanish analysis of the AIRE study. Eur J Heart Fail. 2002;4:553-8.

66. Inomata T, Izumi T, Kobayashi M. Cost-effectiveness analysis of carvedilol for the treatment of chronic heart failure in Japan. Circ J. 2004;68:35-40.

67. Kourlaba G, Parissis J, Karavidas A, et al. Economic evaluation of ivabradine in the treatment of chronic heart failure in Greece. BMC Health Serv Res. 2014;14:631.

68. Pradelli L, Iannazzo S, Zaniolo O. The cost effectiveness and cost utility of valsartan in chronic heart failure therapy in Italy: a probabilistic Markov model. Am J Cardiovasc Drugs. 2009;9:383-92.

69. Ramos IC, Versteegh MM, de Boer RA, et al. Cost effectiveness of the angiotensin receptor neprilysin inhibitor sacubitril/valsartan for patients with chronic heart failure and reduced ejection fraction in the Netherlands: a country adaptation analysis under the former and current Dutch Pharmacoeconomic Guidelines. Value Health. 2017;20:1260-9.

70. Schadlich PK, Huppertz E, Brecht JG. Cost-effectiveness analysis of ramipril in heart failure after myocardial infarction. Economic evaluation of the Acute Infarction Ramipril Efficacy (AIRE) study for Germany from the perspective of Statutory Health Insurance. Pharmacoeconomics. 1998;14:653-69.

71. van Genugten ML, Weintraub WS, Zhang Z, Voors AA. Costeffectiveness of eplerenone plus standard treatment compared with standard treatment in patients with myocardial infarction 
complicated by left ventricular systolic dysfunction and heart failure in the Netherlands. Neth Heart J. 2005;13:393-400.

72. Barry M. Cost effectiveness of beta blocker therapy for patients with chronic severe heart failure in Ireland. Ir Med J. 2002;95(174):6-7.

73. Polistena B, Maggioni AP, Oliva F, Spandonaro F. Cost-effectiveness and budget impact of ivabradine in heart failure therapy. G Ital Cardiol (Rome). 2014;15:626-33.

74. Tilson L, McGowan B, Ryan M, Barry M. Cost-effectiveness of spironolactone in patients with severe heart failure. Ir J Med Sci. 2003;172:70-2.

75. van der Pol S, Degener F, Postma MJ, Vemer P. An economic evaluation of sacubitril/valsartan for heart failure patients in the Netherlands. Value Health. 2017;20:388-96.

76. Yao G, Freemantle N, Flather M, et al. Long-term cost-effectiveness analysis of nebivolol compared with standard care in elderly patients with heart failure: an individual patient-based simulation model. Pharmacoeconomics. 2008;26:879-89.

77. Caro JJ, Migliaccio-Walle K, O'Brien JA, et al. Economic implications of extended-release metoprolol succinate for heart failure in the MERIT-HF trial: a US perspective of the MERIT-HF trial. J Card Fail. 2005;11:647-56.

78. McMurray JJ, McGuire A, Davie AP, Hughes D. Cost-effectiveness of different ACE inhibitor treatment scenarios post-myocardial infarction. Eur Heart J. 1997;18:1411-5.
79. Baio G, Dawid AP. Probabilistic sensitivity analysis in health economics. Stat Methods Med Res. 2015;24:615-34.

80. Bennett JA, Riegel B, Bittner V, Nichols J. Validity and reliability of the NYHA classes for measuring research outcomes in patients with cardiac disease. Heart Lung. 2002;31:262-70.

81. Gibelin P. An evaluation of symptom classification systems used for the assessment of patients with heart failure in France. Eur J Heart Fail. 2001;3:739-46.

82. Raphael C, Briscoe C, Davies J, et al. Limitations of the New York Heart Association functional classification system and self-reported walking distances in chronic heart failure. Heart. 2007;93:476-82.

83. Goldstein BA, Navar AM, Carter RE. Moving beyond regression techniques in cardiovascular risk prediction: applying machine learning to address analytic challenges. Eur Heart J. 2017;38:1805-14.

84. Weng SF, Reps J, Kai J, et al. Can machine-learning improve cardiovascular risk prediction using routine clinical data? PLoS One. 2017;12:e0174944.

85. Strong M, Oakley JE, Brennan A. Estimating multi-parameter partial Expected Value of Perfect Information from a probabilistic sensitivity analysis sample: a non-parametric regression approach. Med Decis Making. 2014;34:311-26. 